\title{
Nonlinear Feedback Control of Spatiotemporal Chaos in Coupled Map Lattices
}

\author{
JIN-QING FANG ${ }^{\mathrm{a}}$ and M.K. ALI ${ }^{\mathrm{b}, *}$ \\ ${ }^{a}$ China Institute of Atomic Energy, P.O. Box 275-27, Beijing 102413, People's Republic of China; ${ }^{\mathrm{b}}$ Department of Physics, \\ The University of Lethbridge, Lethbridge, Alberta T1K 3M4, Canada
}

(Received in final form 5 May 1997)

\begin{abstract}
We describe a nonlinear feedback functional method for study both of control and synchronization of spatiotemporal chaos. The method is illustrated by the coupled map lattices with five different connection forms. A key issue addressed is to find nonlinear feedback functions. Two large types of nonlinear feedback functions are introduced. The efficient and robustness of the method based on the flexibility of choices of nonlinear feedback functions are discussed. Various numerical results of nonlinear control are given. We have not found any difficulty for study both of control and synchronization using nonlinear feedback functional method. The method can also be extended to time continuous dynamical systems as well as to society problems.
\end{abstract}

Keywords: Chaos, Control, Synchronization, Feedback, Nonlinear, Spatiotemporal, Coupled, Map, Lattice

PACS numbers: $05.45+\mathrm{b}, 89.70 .+\mathrm{c}, 43.72 .+\mathrm{q}, 47.52 .+\mathrm{j}$

\section{INTRODUCTION}

"Two fundamental questions dominate future chaos control theories. The first is the problem of controlling higher-dimensional chaos in physical systems. ... The second question has yet to be addressed: the problem of control in a spatiotemporal system" [1]. In recent years, control and synchronization of hyperchaos in higher-dimensional systems as well as spatiotemporal chaos (STC) in spatially extended systems have become a much more important and challenging subject. This is because the behaviors in spatially extended systems are extremely rich [2], such as STC, patterns formation, traveling waves, spiral waves, turbulence and so on. Especially, the control and synchronism of such behaviors have extensive and great potential of interdisciplinary applications, such as security communication, laser, many fluid dynamics, biological systems, crystal growth, information processing, chemical reactor, biochemistry, medicine and engineering. However,

\footnotetext{
* Corresponding author.
} 
this subject is not much known and remains an outstanding open problem.

Spatially extended systems are often modeled by partial differential equations (PDE), ordinary differential equations (ODE), cellular automata (e.g. cellular neural networks (CNNs)) and coupled map lattices (CML). For example, CNN has been found to be very effective for parallel signal processing and real-time simulation of phenomena described by PDE [3]. STC occurs in continuous physical systems (such as in optics and plasma systems, etc.) when different types of motion interact to destroy the spatial coherence of the system concurrent with the onset of temporal chaos. This phenomenon is also described by PDE [4]. Controlling STC in such systems turns out to be more difficult and much more interesting. Here we do not seek to describe the characterization and classification of the all possible spatiotemporal behavior. Our goal in this letter will focus on control of STC by using nonlinear feedback functional method (NFFM). Because the CML, among the above models, has been quite popular recently, various studies have been carried out $\mathrm{o} / \mathrm{n}$ it. The reasons for its popularity are simplicity in analysis and simulation [2]. This model is tractable, easy to handle numerically as well as analytically, and is sometimes able to capture the essential qualitative feature of physical systems. Detailed studies show [2] that it can give rise to a variety of rich spatial and temporal structures, therefore, we will illustrate the NFFM with the CML in this paper.

There have been some attempts toward attaining control and synchronization and offer the possibilities of control of STC in recent years [3-24]. Among them, however, most of above methods are based on linearized models and the concept of linear feedback which is restricted to small perturbation in the linear regime. Therefore this paper is particularly interested in nonlinear feedback methods (e.g. [22-24]). Here we mention some of above methods. An experimental control and synchronization of spatially extended globally coupled multimode laser was carried out [5]. The pinning feedback in both coordinate space and momentum space is used to control in a one-dimensional nonlinear drift-wave equation driven by a sinusoidal wave [6]. A small perturbation feedback control approach is applied to control the uniform field point of STC in the CML with open boundary condition [7]. The above methods are an extension of the OttGrebogi-Yorks method (utilizing linear feedback to stabilize a UPO) and cannot be readily applied to control STC in systems comprising continuously extended media since they are intrinsically globally coupled and infinite-dimensional. They are valid only for limited set of parameter values. The other theoretical study in stabilizing the no-motion state in the RayleighBenard convection was carried out by perturbing the boundary in a feedback manner [8]. A videofeedback control experiment which eliminated chaotic temporal fluctuation of thermal patterns on a catalytic wafer is implemented [14]. Another feedback method is based on the idea of stabilization of unstable periodic patterns (UPP) embedded in STC attractor [4]. It uses a small time- and space-dependent feedback to perturb a variable of the system and has been demonstrated numerically in a transversely extended three-level laser. Although most control methods for the above various spatially extended systems belong to linear feedback, nonlinear control of STC has been paid attention to recently, such as integrated approach for nonlinear feedback control was presented with the Gray-Scott model for cubic autocatalysis in a flow reactor [21]. Therefore this subject still calls for new approaches, in which nonlinear feedback control has become a much more important direction both in automatic control as well as in control chaos [22]. Very recently, we have suggested a method of nonlinear feedback functions (NFF) which has been applied to synchronization of chaos and hyperchaos in higher-dimensional continuous systems as well as spatiotemporal chaos [23].

An interesting question that can be asked is the following: What is the relationship between control and synchronization? Could one give a unified formulation (description) for both cases? In this 
work, on the one hand, a unified description for both control and synchronization is discussed in Section 2 at first. On the other hand, we apply the NFFM to control STC in spatially extended system based on the idea of stabilization of UPP embedded in STC. Both cases have a common subject with different goal (reference) states for their purpose. A good strategy that can be used for both cases is the variable feedback method. As examples, some typical kinds of NFF and their algorithms are introduced for controlling STC in Section 3. We take the various coupled forms of the CML as our models to demonstrate the NFFM through the numerical simulations in Section 4. The main results are shown in Section 5. Finally, discussions and conclusions are provided.

\section{A UNIFIED DESCRIPTION FOR STUDY OF BOTH CONTROL AND SYNCHRONIZATION}

In this section, we would like to describe a unified formulation for both control and synchronization of STC (including chaos and hyperchaos). Because synchronization method of the NFF can be easily extended to control of STC once the relationship between control and synchronization of chaos is clarified. In our point of view, control and synchronization have a common basis with different reference states and end results. A good strategy that can be used for both cases is to apply the linear and nonlinear variable feedback method. We now pay attention to nonlinear feedback control since most of previous works are based on the linear feedback. Let us consider, for illustration, the coupled map lattice (CML) which can be defined as:

$$
\begin{gathered}
X_{n+1}(i)=\beta_{i} F\left(X_{n}(i)\right)+\frac{1}{\mu} \sum_{j=1, j \neq i}^{\mu} \epsilon_{i j} h\left(X_{n}\left(m_{i j}\right)\right), \\
i=1,2, \ldots, L, \quad \mu \leq L-1,
\end{gathered}
$$

where $F\left(X_{n}(i)\right)$ describes the internal dynamics of the $i$ th site when couplings with other sites are absent. For example, we take $F\left(X_{n}\right)=a X_{n}\left(1-X_{n}\right)$ which is the one-dimensional logistic map behaving chaotically in the range of $a=3.8-4$. In Eq. (1), $i$ and $n$ denote discrete space and time variables and $L$ is the total number of sites each of which is connected to $\mu$ neighbors, $\beta_{i}$ is $i$ th system parameter, $m_{i j}$ is the index of the $j$ th neighbor of the $i$ th site, $\epsilon_{i j}$ is the coupling strength of the $j$ th neighbor of the $i$ th site and $h\left(X_{n}\left(m_{i j}\right)\right)$ is a function of the state variables $X_{n}\left(m_{i j}\right)$. The dynamical behavior of a CML depends on the parameters $\beta_{i}$, the nature of coupling (type of $h$ and values of $m_{i j}$ ), the coupling strengths $\epsilon_{i j}$ and boundary conditions. We have used periodic boundary conditions in this work. Using the feedback functions

$$
\begin{aligned}
& \mathbf{G}_{n}(\mathbf{X}, \mathbf{Y})=\sum_{j=1}^{\nu} \alpha_{i j} g_{n}\left(X_{n}\left(m_{i j}\right), Y_{n}\left(m_{i j}\right)\right), \quad \nu \leq L \\
& \quad i=1,2, \ldots, L
\end{aligned}
$$

the controlled/synchronized CML then becomes

$$
\begin{aligned}
Y_{n+1}(i)= & \beta_{i} F\left(Y_{n}(i)\right)+\frac{1}{\mu} \sum_{j=1, j \neq i}^{\mu} \epsilon_{i j} h\left(Y_{n}\left(m_{i j}\right)\right) \\
& +\mathbf{G}_{n}(\mathbf{X}, \mathbf{Y})
\end{aligned}
$$

where $i=1,2, \ldots, L, \mu \leq L-1$. Equations (1) and (3) are also called the drive, master and response, slave systems, respectively, if we consider synchronization between Eq. (1) and Eq. (3) with $\mathbf{Y}(\mathbf{0}) \neq \mathbf{X}(\mathbf{0})$. Therefore controlled and synchronized systems can be unified by the same formulation, Eqs. (1)-(3). If a desired unstable period orbits (UPO) or UPP embedded in strange STC attractor is stabilized, i.e., the state vectors $\mathbf{X}_{n}=\mathbf{X}_{\mathrm{d} n}$ control is achieved. For synchronization between the master (Eq. (1)) and slave systems (Eq. (3)) it is said to be achieved if $\mathbf{Y}=\mathbf{X}$, where $\mathbf{X}$ is (spatiotemporal or temporal) chaotic reference (goal) state. In other words, Eqs. (1)-(3) can describe both the problems, of controlling and synchronizing spatiotemporal chaos, of course including chaos as well as hyperchaos 
[23]. A common important property of the feedback function for both cases is that it vanishes when synchronization or control is realized. For discrete map systems, that is

$$
\mathbf{G}_{n}\left(\mathbf{X}_{n}, \mathbf{X}_{\mathrm{d} n}\right)=0 \quad \text { when } \mathbf{X}_{n}=\mathbf{X}_{\mathrm{d} n},
$$

or for continuous systems

$$
\mathbf{G}(\mathbf{X}, \mathbf{Y})=\mathbf{G}(\mathbf{X}, \mathbf{X})=0 \quad \text { when } \mathbf{Y}=\mathbf{X} \text {. }
$$

The state vector $\mathbf{X}_{n}\left(\mathbf{X}_{n}=\mathbf{X}_{\mathrm{d} n}\right)$ in the controlled system describes the desired or 'goal' UPO/UPP if the STC is synchronized to $\mathbf{X}_{\mathrm{d} n}$ in the CML. In the following CML, we often use $\mathbf{G}_{n}(i)$ for the CML instead of $\mathbf{G}_{n}(\mathbf{X}, \mathbf{Y})$, i.e., $\mathbf{G}_{n}(i)=\mathbf{G}_{n}\left(\mathbf{X}_{n}, \mathbf{X}_{\mathrm{d} n}\right)$. The condition of control and synchronization can be satisfied by a large class of functional forms of $\mathbf{G}_{n}(i)$ or $\mathbf{G}(\mathbf{X}, \mathbf{Y})$. Some simple linear forms of $\mathbf{G}(\mathbf{X}, \mathbf{Y})$ have been used previously. For example,

$$
\begin{aligned}
& \mathbf{G}_{i}(\mathbf{X}, \mathbf{Y})=K_{i}\left(Y_{i}-X_{i}\right), \\
& \mathbf{G}_{i}(\mathbf{X}, \mathbf{Y})=B_{i} \sum_{j=1}^{n} K_{j}\left(Y_{j}-X_{j}\right) ; \\
& \quad i=1,2, \ldots, n .
\end{aligned}
$$

Equations (6) and (7) contain $n$ and $n^{2}$ feedback parameters $K_{i}$ and $B_{i}$ respectively. $\mathbf{K}$ is matrix of the feedback parameters. However, control or synchronization may be achieved by a fewer number of parameters: in some cases control/synchronization is possible even by adjusting only one parameter. We have shown that the NFFM are effective for synchronizing chaotic as well as hyperchaotic systems [23]. The method will be extended further to control of STC in this work. The important point to note here is that the flexibility in the choice of $\mathbf{G}_{n}(i)$ or $\mathbf{G}(\mathbf{X}, \mathbf{Y})$ provides robustness and efficiency to the method for dealing with not only chaotic and hyperchaotic, but also spatiotemporal chaotic systems of diverse nature.

The transition time of realizing control/ synchronization for a given CML is measured by the zeros (to machine precision) of the function
$\Delta(\cdot)$, where for discrete map systems

$$
\Delta(n)=\sqrt{\sum_{i=1}^{L}\left(X_{n}(i)-X_{\mathrm{d} n}(i)\right)^{2}}
$$

and for continuous systems,

$$
\Delta(t)=\sqrt{\sum_{i=1}^{L}(Y-X)^{2}} .
$$

This is a qualifying measure for both control and synchronization.

The above unified description for study of both control and synchronization in the CML can be easily extended to continuous spatially extended systems. To do this, let us consider two $n$-dimensional autonomous dynamical systems described by the differential equations

$$
\begin{aligned}
\dot{\mathbf{X}} & =\mathbf{F}(\mathbf{X}), \\
\dot{\mathbf{Y}} & =\mathbf{F}(\mathbf{Y})+\mathbf{G}(\mathbf{X}, \mathbf{Y}) .
\end{aligned}
$$

Here the state vectors $\mathbf{X}, \mathbf{Y} \in R^{n}$ are $n$-dimensional vectors with components $X_{1}, X_{2}, \ldots, X_{n}$ and $Y_{1}, Y_{2}, \ldots, Y_{n}$ respectively. The vector functions $\mathbf{F}$ and $\mathbf{G}$ have the components $F_{1}, F_{2}, \ldots, F_{n}$ and $G_{1}, G_{2}, \ldots, G_{n}$ respectively. The Eqs. (8) and (9) are equivalent to Eqs. (1) and (3). The function $\mathbf{F}(\mathbf{Y})$ may be a replica of the function $\mathbf{F}(\mathbf{X})$ but may be a different one from the point of view of generalized synchronization/control. $\mathbf{G}(\mathbf{X}, \mathbf{Y})$ is the above feedback function. For control we only use Eq. (9) and is enough to study. For synchronization, however, the dynamics of the $\mathbf{X}$ and $\mathbf{Y}$ systems are needed and started from different initial conditions $(\mathbf{Y}(t=0) \neq \mathbf{X}(t=0))$. Control and synchronization are said to be achieved if the dynamical system describing the time evolution of the difference $\mathbf{e}=\mathbf{Y}-\mathbf{X}$, i.e, the error equation

$$
\begin{aligned}
\dot{\mathbf{e}} & =\mathbf{F}(\mathbf{Y})+\mathbf{G}(\mathbf{X}, \mathbf{Y})-\mathbf{F}(\mathbf{X}) \\
& =\mathbf{F}(\mathbf{X}+\mathbf{e})+\mathbf{G}(\mathbf{X}, \mathbf{X}+\mathbf{e})-\mathbf{F}(\mathbf{X})
\end{aligned}
$$

has a stable fixed point at $\mathbf{e}=0$. Another way of saying this is that we achieve control/ 
synchronization if $\|\mathbf{Y}-\mathbf{X}\| \rightarrow 0$ and $\mathbf{G}(\mathbf{X}, \mathbf{Y}) \rightarrow 0$ as $t \rightarrow \infty$, where $\mathbf{X}$ is a desired (goal) state. The above control or synchronization conditions can be satisfied by a very large number of linear and nonlinear functional forms of $\mathbf{G}(\mathbf{X}, \mathbf{Y})$. The feedback scheme of Eqs. (1) and (3) and Eqs. (8) and (9) not only includes the approaches in current references and our suggestions in the next section but also has the flexibility of introducing all possible new feedback functions and thus giving robustness to the method of feedback control. For example, recently, Kocarev and Parlitz have used an active-passive decomposition scheme for synchronization [24]. This scheme involves decomposing the dynamical systems as:

$$
\begin{aligned}
\dot{\mathbf{X}} & =\mathbf{F}(\mathbf{X}, \mathbf{s}), \\
\dot{\mathbf{Y}} & =\mathbf{F}(\mathbf{Y}, \mathbf{s}), \\
\mathbf{s} & =\mathbf{h}(\mathbf{X}), \\
\dot{\mathbf{s}} & =\mathbf{h}(\mathbf{X}, \mathbf{s}) .
\end{aligned}
$$

Mathematically, this decomposition scheme is included in our approach if we choose $\mathbf{G}(\mathbf{X}, \mathbf{Y})=$ $\mathbf{F}(\mathbf{Y}, \mathbf{s})-\mathbf{F}(\mathbf{Y})$. The mathematical equivalence between their decomposition scheme and the approaches of Pecora and Carroll [25] has been shown. Therefore, the feedback scheme of Eqs. (8) and (9) or Eqs. (1)-(3) is of universal formulation which includes control approaches as well as synchronization. The evidences are that a dynamical system can be synchronized by a variety of choices of the feedback function $\mathbf{G}(\mathbf{X}, \mathbf{Y})$ [23], as shown later on. A key issue addressed is how to find all families of nonlinear feedback functions NFF from a general method. One of the important points to find NFF is based on their property of $\mathbf{G}_{n}(i)$ or $\mathbf{G}(\mathbf{X}, \mathbf{Y})$, which tends to zero and has a well-behaved (no intermittence and no catastrophe for long time and so on) when control/synchronization is achieved for long time. Some feedback functions for a given system (e.g. CMLs) are sampled in next section by trial and error but a general method of generating NFF is still open.

\section{NONLINEAR FEEDBACK FUNCTIONS AND CONTROL ALGORITHMS}

Now we are in a position to discuss the key issue of the method. In spatially extended systems, there may exist a large number of UPP states even in the presence of a stable output. Consider the two cases: (1) in the first case assume that some UPO or UPP states are known and (2) in the second case exist unknown UPO/UPP states. In view of these two cases, we would like to classify the NFF into two kinds. The first type is one for stabilizing desired UPO or UPP embedded in the original chaotic/STC attractor. Second type is one for generating certain new dynamics that we need. In other words, there are two types of NFF to be applied to control and synchronize spatiotemporal chaos. For the first one, NNF1, an important property of the feedback functions is that they vanish $\left(\mathbf{G}_{n}(i)=0\right)$ when control is realized. Because these NFF1 can be designed to stabilize or synchronize any desired UPO or UPP embedded in the STC. Therein the NFF2, $\mathbf{G}_{n}(i)$ does not vanish, it is designed not only to stabilize UPO or UPP but also to generate new patterns one expects. In any case, two types of NFF are not unique, i.e., there are many different families of feedback functions that can control/synchronize a goal state or generate a desired new dynamics. A large variety of feedback functions can be found as illustrated below. For the NFF1 corresponding to logistic map, we have:

$$
\begin{aligned}
& g_{n}(i)=\left(X_{\mathrm{d} n}(i)-X_{n}(i)\right)\left(2 X_{n}(i)-1\right), \\
& g_{n}(i)=\frac{1}{2}\left(X_{\mathrm{d} n}(i)-X_{n}(i)\right)\left(k X_{n}(i)^{2}-1\right), \\
& k=3,4, \ldots, \\
& g_{n}(i)=\tanh \left[\left(X_{\mathrm{d} n}(i)-X_{n}(i)\right)\left(2 X_{n}(i)-1\right)\right], \\
& g_{n}(i)=\left(X_{\mathrm{d} n}(i)-X_{n}(i)\right) \tanh \left[\left(2 X_{n}(i)-1\right)\right], \\
& g_{n}(i)=\left(2 X_{n}(i)-1\right) \tanh \left(X_{\mathrm{d} n}(i)-X_{n}(i)\right), \\
& g_{n}(i)=\left(2 X_{n}(i)-1\right) \sin \left(X_{\mathrm{d} n}(i)-X_{n}(i)\right),
\end{aligned}
$$




$$
\begin{aligned}
& g_{n}(i)=\sin \left[\left(X_{\mathrm{d} n}(i)-X_{n}(i)\right)\left(2 X_{n}(i)-1\right)\right], \\
& g_{n}(i)=\left(X_{\mathrm{d} n}(i)-X_{n}(i)\right) \sin \left[\left(2 X_{n}(i)-1\right)\right],
\end{aligned}
$$

etc.,

where $X_{\mathrm{d} n}(i)$ is a desired (known) UPO or UPP in the CML. The first kind NFF1 is suitable for controlling UPO/UPP of the CMLs known either from experiment or numerical calculation/analysis [26]. How to construct such NFF1 is the most important problem. One of the key points is based on the property of $\mathbf{G}_{n}(i)$ which tends to zero and is well-behaved (no intermittence and no catastrophe for long time, and so on) when control or synchronization is achieved for long time. A construction method for feedback functions is Lyapunov functional one which will be given elsewhere. So there are large classes of the NFF1s which may satisfy the above property. It has been demonstrated that the above $g_{n}(i)$ forms (Eqs. (15)-(22)) can be applied to realize synchronization of chaos, hyperchaos and spatiotemporal chaos if it is all right in the parameter space [23]. The main idea of the control algorithms corresponding to the above NNF1 is that a nonlinear feedback (term) signal, $\mathbf{G}_{n}(i)$ corresponding to NFF1, is assigned to perturb the spatial dynamical variables at each site or sporadic sites directly. The NFF1 is designed for the controlled (or response) system to follow (or synchronize with) the desired UPP embedded in the STC attractor. Therefore the NFF1 tends to zero when the control/synchronization is achieved. In other words, the nonlinear signal forces the response system to be synchronized with one desired UPP/UPO.

For the second one, NFF2 corresponding to logistic map, we have:

$$
\begin{aligned}
& g_{n}(i)=K_{i} X_{n}(i)\left(\lambda_{i} A_{i} X_{n}(i)-X_{\mathrm{d} n}(i)\right) \\
& g_{n}(i)=K_{i}\left(X_{n}(i)-X_{\mathrm{d} n}(i)\right)\left(\lambda_{i} X_{n}(i)-1\right) \\
& g_{n}(i)=A_{i} \sin \lambda_{i}\left(X_{n}(i)-X_{\mathrm{d} n}(i)\right) \\
& g_{n}(i)=1-B_{i} \cos \lambda_{i}\left(X_{n}(i)-X_{\mathrm{d} n}(i)\right) \\
& g_{n}(i)=B_{i}\left(1-\cos \lambda_{i}\left(X_{n}(i)-X_{\mathrm{d} n}(i)\right)\right)
\end{aligned}
$$

$$
\begin{aligned}
g_{n}(i)= & K_{i} X_{n}(i)\left(X_{n}(i)-X_{\mathrm{d} n}(i)\right) \\
& +A_{i} \sin \lambda_{i}\left(X_{n}(i)-X_{\mathrm{d} n}(i)\right) \\
\text { etc., } &
\end{aligned}
$$

where $A_{i}, B_{i}, K_{i}$ and $\lambda_{i}, i=1,2, \ldots, n$ are adjustable parameters. The NFF2 is suitable for unknown UPO/UPP in the master systems when one wants to get certain stable new pattern state. The above forms of $g_{n}(i)$, Eqs. (23)-(28), can control STCs to generate some of the desired patterns for Eq. (3) or Eq. (9). The results will also be given below. Other forms of the NFF, both NFF1 and NFF2, are conceivable. In the following section, we consider CMLs for which we have successfully controlled STC by the NFFM.

\section{FIVE CONNECTION FORMS FOR THE CML}

Let us now consider various coupled forms for the CML in order to test the efficiency of the NFFM. With a view to possible applications to neural networks, we are particularly interested in (i) lattices with random neighbors and fixed coupling strength (CMLR) and (ii) lattices with random neighbors and random coupling strengths (CMLRR). For the CMLR and CMLRR, the $m_{i j}$ were found by a random number generator and we set $h\left(X_{n}\left(m_{i j}\right)\right)=F\left(X_{n}\left(m_{i j}\right)\right)$. The CMLR and CMLRR are, respectively, given under the control by:

$$
\begin{aligned}
X_{n+1}(i)= & \gamma\left[(1-\epsilon) f_{n}(i)+\frac{\epsilon}{\nu} \sum_{j=1, j \neq i}^{\nu} f_{n}\left(m_{i j}\right)\right] \\
& +\mathbf{G}_{n}(i), \\
X_{n+1}(i)= & \gamma\left[(1-\epsilon) f_{n}(i)+\frac{1}{\nu} \sum_{j=1, j \neq i}^{\nu} \alpha_{i j} f_{n}\left(m_{i j}\right)\right] \\
& +\mathbf{G}_{n}(i),
\end{aligned}
$$

while for the types of CMLs described by some authors, such as Kaneko [2], Shinbrot [18] and $\mathrm{Hu}$ [6] which we call as CMLK, CMLS and 
CMLH respectively, we have:

$$
\begin{aligned}
X_{n+1}(i)= & \gamma\left[(1-\epsilon) f_{n}(i)+\frac{\epsilon}{L} \sum_{j=1}^{L} f_{n}(j)\right]+\mathbf{G}_{n}(i), \\
X_{n+1}(i)= & \gamma\left[(1-\epsilon) f_{n}(i)\right. \\
& \left.+\frac{\kappa}{L-1} \sum_{j=1, j \neq i}^{L-1}\left(X_{n}(j)-Y_{n}(j)\right)\right]+\mathbf{G}_{n}(i), \\
X_{n+1}(i)= & \gamma\left[(1-\epsilon) f_{n}(i)\right. \\
& +\frac{\epsilon}{2 N} \sum_{j=1}^{N}\left(f_{n}(i+j)+f_{n}(i-j)\right]+\mathbf{G}_{n}(i),
\end{aligned}
$$

and their corresponding parameters for Eq. (1) are as follows:

$$
\begin{aligned}
& \beta_{i}=1+\frac{(1-L) \epsilon}{L}, \quad \epsilon_{i j}=\epsilon, \quad \mu=L-1, \\
& \beta_{i}=p, \quad \mu=L-1, \quad \frac{\epsilon_{i j}}{\mu}=\frac{\kappa}{L-1}, \\
& \beta_{i}=1-\epsilon, \quad \frac{\epsilon_{i j}}{\mu}=\frac{\epsilon}{2 N},
\end{aligned}
$$

where $\gamma$ is an adjustable parameter. The $\alpha_{i j}$ in Eq. (30) for the CMLRR are random numbers with $0 \leq \alpha_{i j} \leq \epsilon$ and the symmetry condition $\alpha_{i j}=\alpha_{j i}$ is not invoked. During the iterations if $X_{n+1}(i)$ falls outside the basin of attraction (here the interval $(0,1))$, then the contribution of the feedback term is scaled so that $X_{n+1}(i)$ belongs to this interval or simply set $X_{n+1}(i)=\left|X_{n+1}(i)\right|$ modulo 1. As example, some common parameters of the CMLs we used in this text are as follows: $L=50$ and $a=4$. For control of STC, the NFF1 signal (term), $\mathbf{G}_{n}(i)$, may be applied to every site and at every iteration or it can be applied sporadically. Suppose that solutions of desired UPO/ UPP, $X_{\mathrm{d} n}$, are specified for Eqs. (29)-(33) in such a way that $\mathbf{G}_{n}(i)$ corresponding to $g_{n}(i)$, tends to vanish when control of the STC is achieved. In other words, the STC can be synchronized to the desired UPO/UPP. The number of neighbors $\nu$ in Eqs. (29) and (30) varied from 1 to $L-1$. It may be pointed out that we can find low periodic solutions for the CMLH, CMLR, CMLK and CLMS analytically or numerically [26]. Thus the NFF1 with any $g_{n}(i)$ from Eqs. (15)-(22) are always found to control and synchronize STC in all of Eqs. (29)-(33), as given later on. For the CMLRR, however, it is rather difficult or is not necessary to find the solutions of UPP analytically or numerically. In such a case, the NFF2 would be suitable for control.

\section{VARIOUS CONTROL RESULTS}

The main results will focus on control by using NFF1 since synchronization of chaos and hyperchaos as well as of STC have been shown mainly in [23]. However, both control and synchronization as two strategic ways are used alternatively for control of STC in this section.

\subsection{Periods in Time and Fixed Point in Space for the CMLR, CMLH and CMLK}

It is interesting to stabilize any desired UPO/UPP of CMLs since there exist numerous UPO/UPP in the CMLs. The dynamics of a CML can be controlled to synchronize with desired periodic orbits by using the above NFF1. Here we only present some examples of UPO/UPP that we have considered. All the periodic solutions are obtained by appropriately choosing the initial values of the coordinates $X_{1}(i), i=1,2, \ldots, L$. Periodic solution with fixed point in space $\left(X_{1}(i)=X_{1}(j), \forall i, j\right)$ and fixed point (FP) in time $\left(\mathbf{X}_{n+1}=\mathbf{X}_{n}, \forall n\right)$ can be found for the CMLR, CMLK and CMLH by using the periodic orbits of the single logistic map, because periodic orbits of the logistic map are easy to find [26]. Some examples of periodic orbits of the logistic map $\left(X_{n+1}=a X_{n}\left(1-X_{n}\right)\right)$ are given below. 
1. Period-2 periodic points:

$$
\begin{aligned}
& X_{1}=\frac{1}{2 a^{2}}\left(a^{2}+a+\sqrt{a^{4}-2 a^{3}-3 a^{2}}\right), \\
& X_{1}=\frac{1}{2 a^{2}}\left(a^{2}+a-\sqrt{a^{4}-2 a^{3}-3 a^{2}}\right) .
\end{aligned}
$$

2. Period-3 periodic points if $a=4$ :

$$
\begin{array}{r}
X_{1}=0.9504844339512096, \\
0.1882550990706332,
\end{array}
$$

or

$$
\text { 0.6112604669781572; }
$$

$$
\begin{aligned}
X_{1}= & 0.9698463103929542, \\
& 0.116977778440511,
\end{aligned}
$$

or

$$
0.4131759111665348 \text {. }
$$

3. Period-5 periodic points if $a=4$ :

$$
\begin{aligned}
X_{1}= & 0.00903565136864665, \\
& 0.03581603349196369, \\
& 0.1381329809474649, \\
& 0.4762090420881289,
\end{aligned}
$$

or

$$
0.9977359612865423 \text {. }
$$

As a typical example, Fig. 1 gives the period-3 in time and FP in space for the CMLR. Following coordinates of 3-dimensional figures is the same as Fig. 1. For the CMLS with $a=4$, the FP and period-2 solutions are obtained by using $X=1 / 4(3+\epsilon)$ and $X_{1}=1 / 8\left(\epsilon+5 \pm \sqrt{\epsilon^{2}+6 \epsilon+5}\right)$ respectively for $X_{1}(i)=X_{1}(j)=X, \forall i, j$. Similar controls for the other CMLs are easily realized.

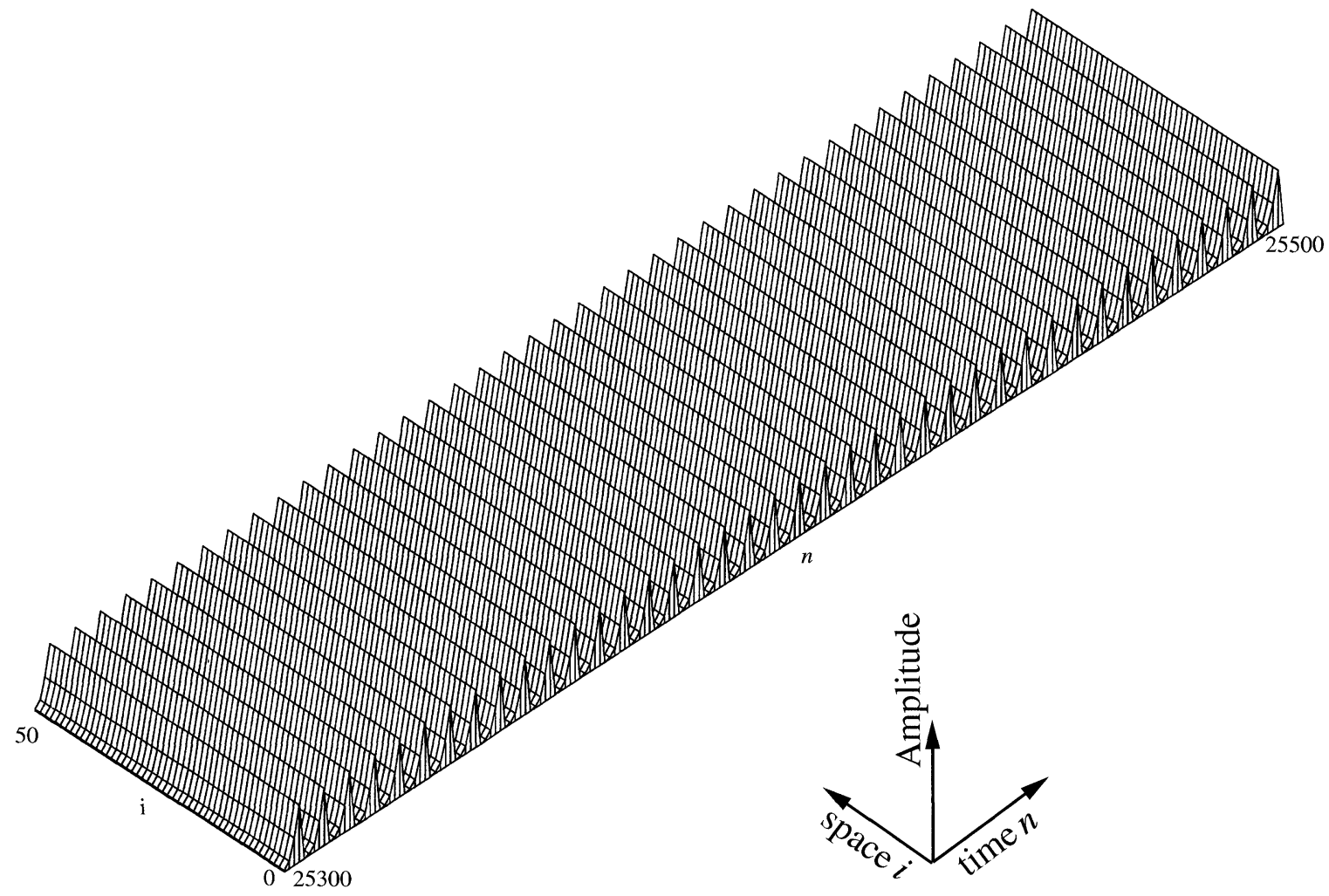

FIGURE 1 The period-3 in time and fixed point in space for the CMLH is stabilized using the NFF1 of Eq. (15). $L=50$, $N=2$ and $\epsilon=0.8$ and $a=4$. Following coordinates of 3-dimensional figures is the same as Fig. 1 . 


\subsection{Stabilize Spatiotemporal Periodic} Solutions by Synchronization Method

A detailed study of spatially periodic solution of CMLs type lattices with nearest neighbor interactions can be found in [26]. In what follows we give examples.

1. Period-2 both in space and in time if $a=4$, and $\epsilon \leq 1 / 4$ :

$$
\begin{aligned}
& X_{1}(1)=\frac{8 \epsilon-5+\sqrt{32 \epsilon^{2}-28 \epsilon+5}}{8(2)(\epsilon-1)}, \\
& X_{1}(2)=\frac{8 \epsilon-5-\sqrt{32 \epsilon^{2}-28 \epsilon+5}}{8(2)(\epsilon-1)},
\end{aligned}
$$

where $\epsilon \leq 1 / 4$ with $X_{1}(j)=X_{1}(1)$ if $j$ is odd and $X_{1}(j)=X_{1}(2)$ if $j$ is even. A period-2 both in space and time can also be obtained by another complicated formula involving $\epsilon$. For example with $\epsilon=0.1$,

$$
X_{1}(1)=0.3747722408220005
$$

and

$$
X_{1}(2)=0.7842654775396390
$$

can generate periodic solutions. Figure 2 shows stabilized period-2 pattern by NFF1 (15).

2. A period-5 in space and period- 2 in time if $a=4$ and $\epsilon=0.1$ :

$$
\begin{aligned}
& X_{1}(1)=0.3908791844931709, \\
& X_{1}(2)=0.9080286804380671, \\
& X_{1}(3)=0.74571288644516, \\
& X_{1}(4)=0.3704760085054254, \\
& X_{1}(5)=0.7843770872390666
\end{aligned}
$$

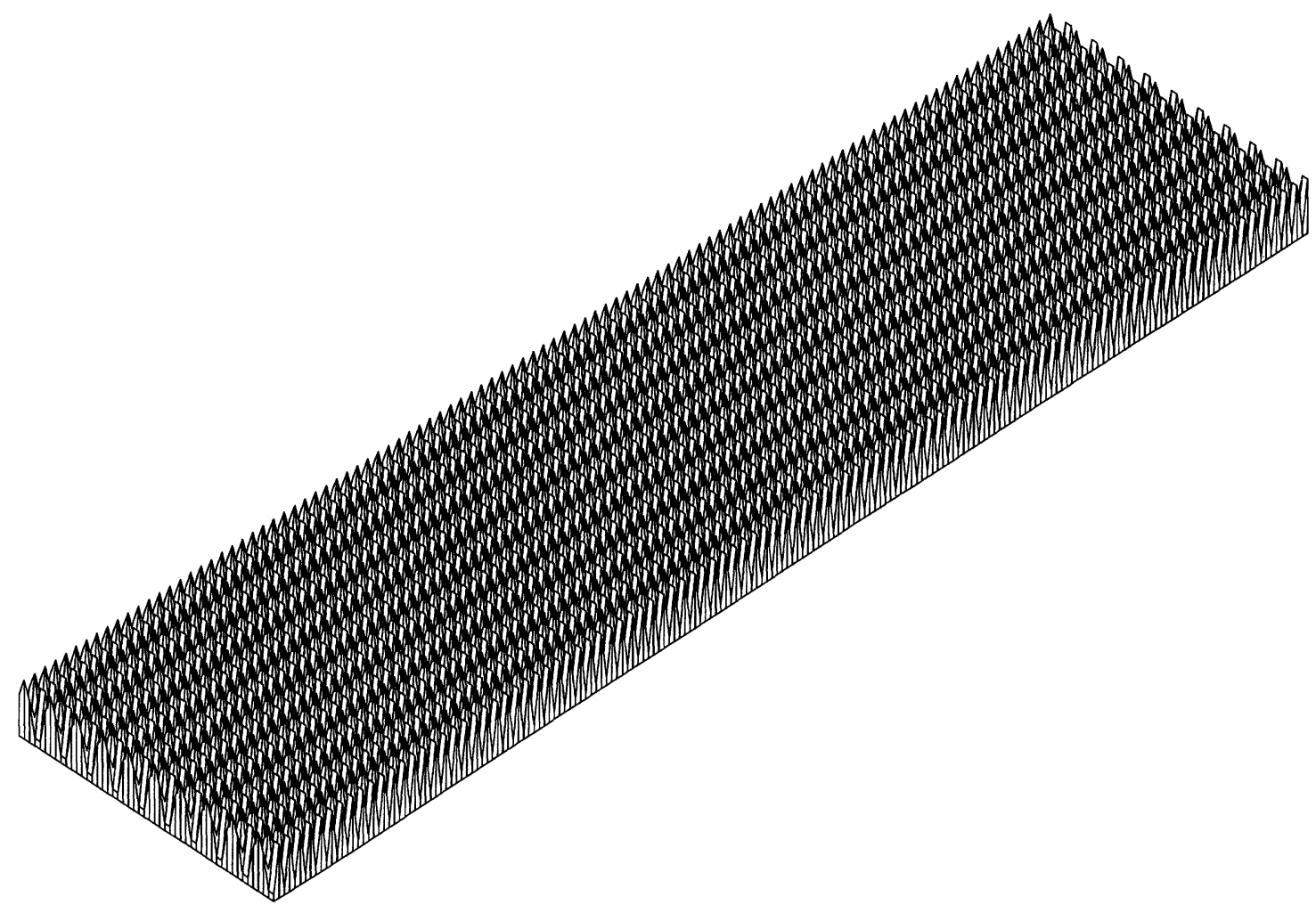

FIGURE 2 The period-2 both in space and time for the CMLS is stabilized using the NFF1 of Eq. (22). $L=50, N=2$ and $\epsilon=0.023$ and $a=4$. 


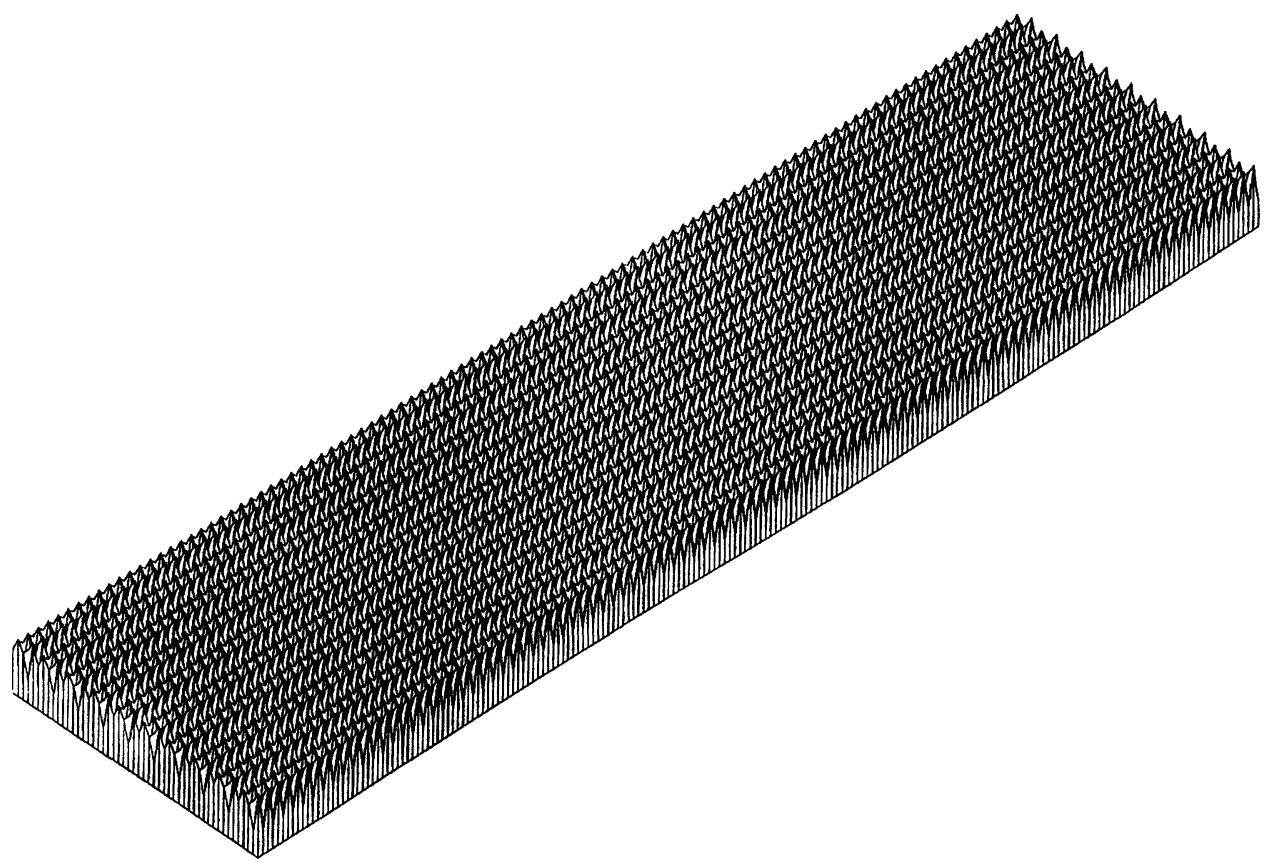

FIGURE 3 The period-5 in space and period-2 in time for the CMLH is stabilized using the NFF1 of Eq. (16) with $K=3$. $L=50, N=2$ and $\epsilon=0.8$ and $a=4$.

with repeating $X_{1}(j)$ after every 5 sites $\left(X_{1}(1)\right.$, $\left.X_{1}(2), X_{1}(3), X_{1}(4), X_{1}(5), X_{1}(1), X_{1}(2), \ldots\right)$. Such a UPP can be also stabilized by the NFF1 Eq. (9), as shown in Fig. 3.

\subsection{Stabilize the Fixed Point both in Space and in Time}

The best test for the NFF1 is to stabilize the fixed-point (FP) solution $X_{f}(i)$ both in space $i$ and in time $n$ for Eq. (1). $X_{f}(i)=(a-1) / a$ is just the unstable FP solution of a single logistic map. If $a=4, X_{f}(i)=0.75$ which is unstable. The FP is easily stabilized for the CMLR, CMLH and CMLK by use of the NFF1 above. However, the FP for the CMLS is: $X_{f}(i)=(3+\epsilon) / 4$. We only take an example of a typical STC in Fig. 4(a) for the CMLRR with $L=50, N=20, \epsilon=0.8$ and $a=4$. The other STCs are similar to the above. The FP is easily stabilized when one of Eqs. (15)-(22) is applied to Eqs. (29)-(33), respectively, as shown in Fig. 4(b) by using NFF1 of $g_{n}(i)=$ $0.7 \sin \left[\left(X_{f}(i)-X_{n}(i)\right)\left(2 X_{n}(i)-1\right)\right]$.

In order to compare the efficiency of NFF1, transition time, $\Delta(n)$, which tends to zero for stabilizing the FP in various CMLs, is given in Figs. 5(a)-(d) for the CMLR, CMLRR, CMLH and CMLK under the control by each same forms of NFF1, respectively. It is seen from Fig. 5 that each of the four kinds of NFF1, Eqs. (8), (16), (14) and (21) can always control the STC in four kinds of connection form of CML. We see the transition time $n$ is generally less than 20 for CMLRR, CMLS and CMLK, and $n=46-66$ for the CMLH.

Figure 6(a)-(d) show the other comparison of transition time $\Delta(n)$ of stabilizing the FP for each of the four coupled forms of CMLs (with common $L=50$ and $a=4$ ) under four kinds of NFF1. In Fig. 2, (a) corresponds to CMLRR (with $N=20, \gamma=4, \epsilon=0.1,0 \leq \alpha_{i j} \leq \epsilon$ ), (b) to CMLS (with $N=2, \epsilon=0.023$ ), (c) to CMLH (with $N=L, \epsilon=0.8$ ) and (d) to CMLK (with 

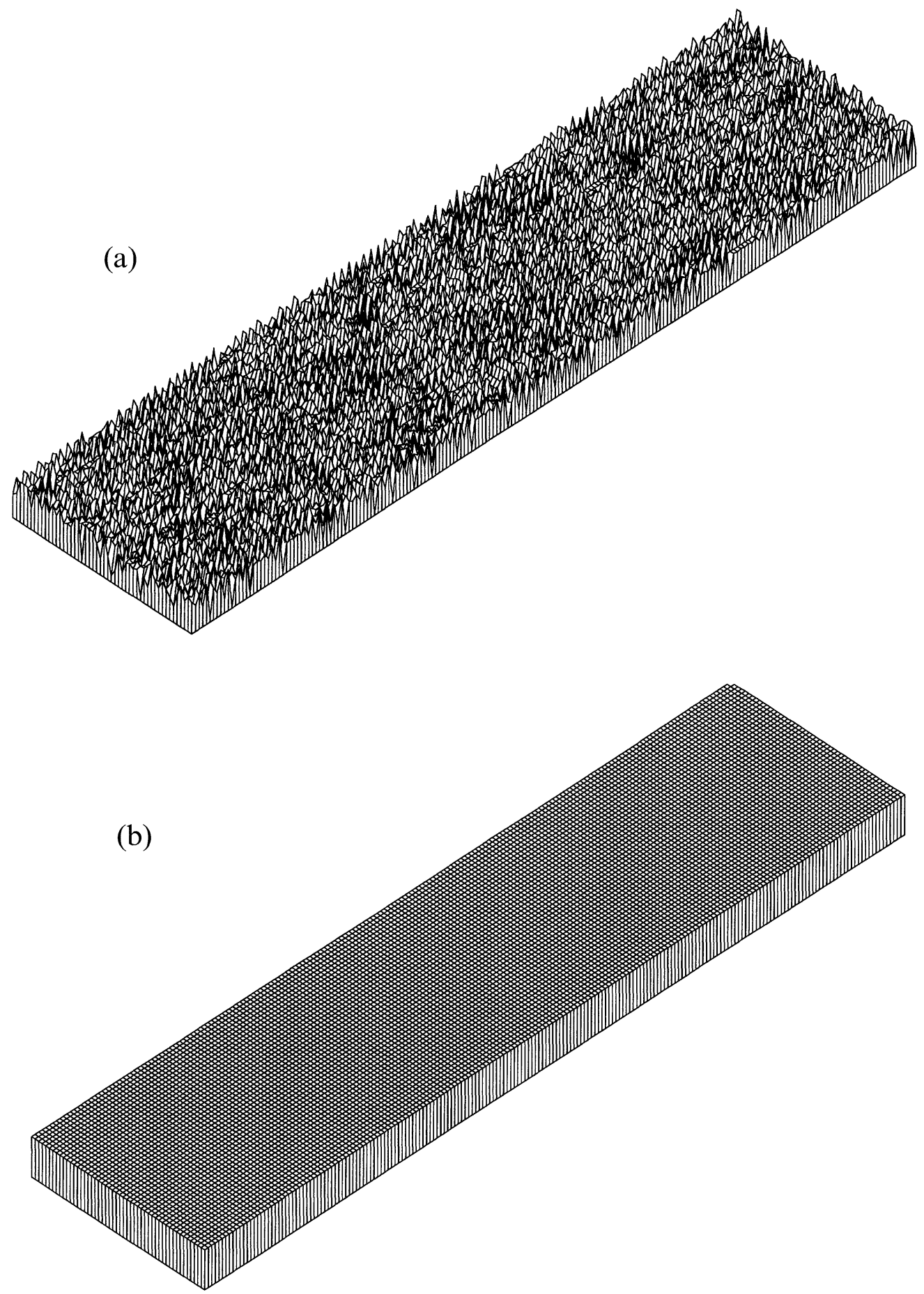

FIGURE 4 A comparison of spatiotemporal chaos without any control with controlled one for the CMLRR by the NFF1. $L=50, N=20$ and $\epsilon=0.3$ and $a=4$. (a) A typical STC in the above CMLRR as a function of discrete space and time variables $i$ and $n$ without any control. (b) The FP is stabilized in the above CMLRR with $\gamma=4$ by using $g_{n}(i)=$ $0.7 \sin \left[\left(X_{f}(i)-X_{n}(i)\right)\left(2 X_{n}(i)-1\right)\right]$. 


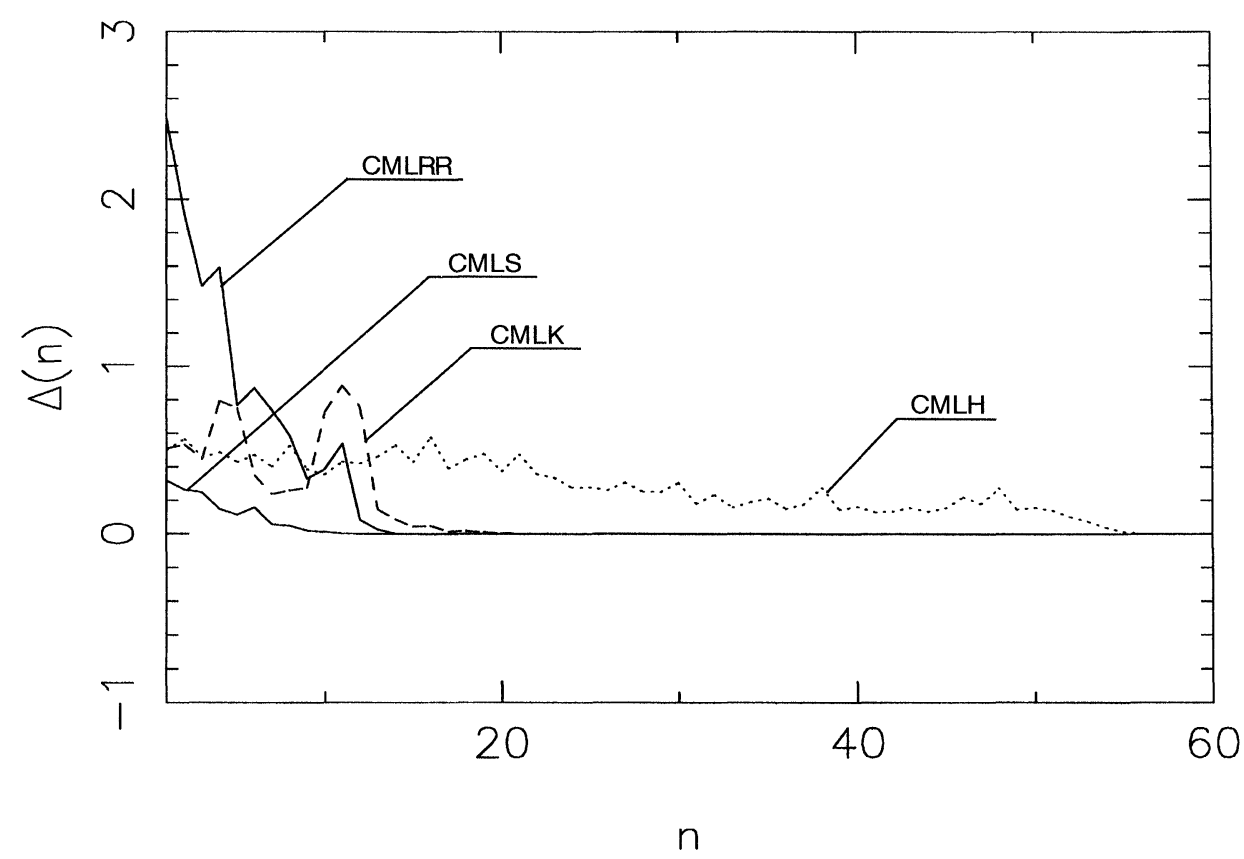

FIGURE 5(a)



FIGURE 5(b) 


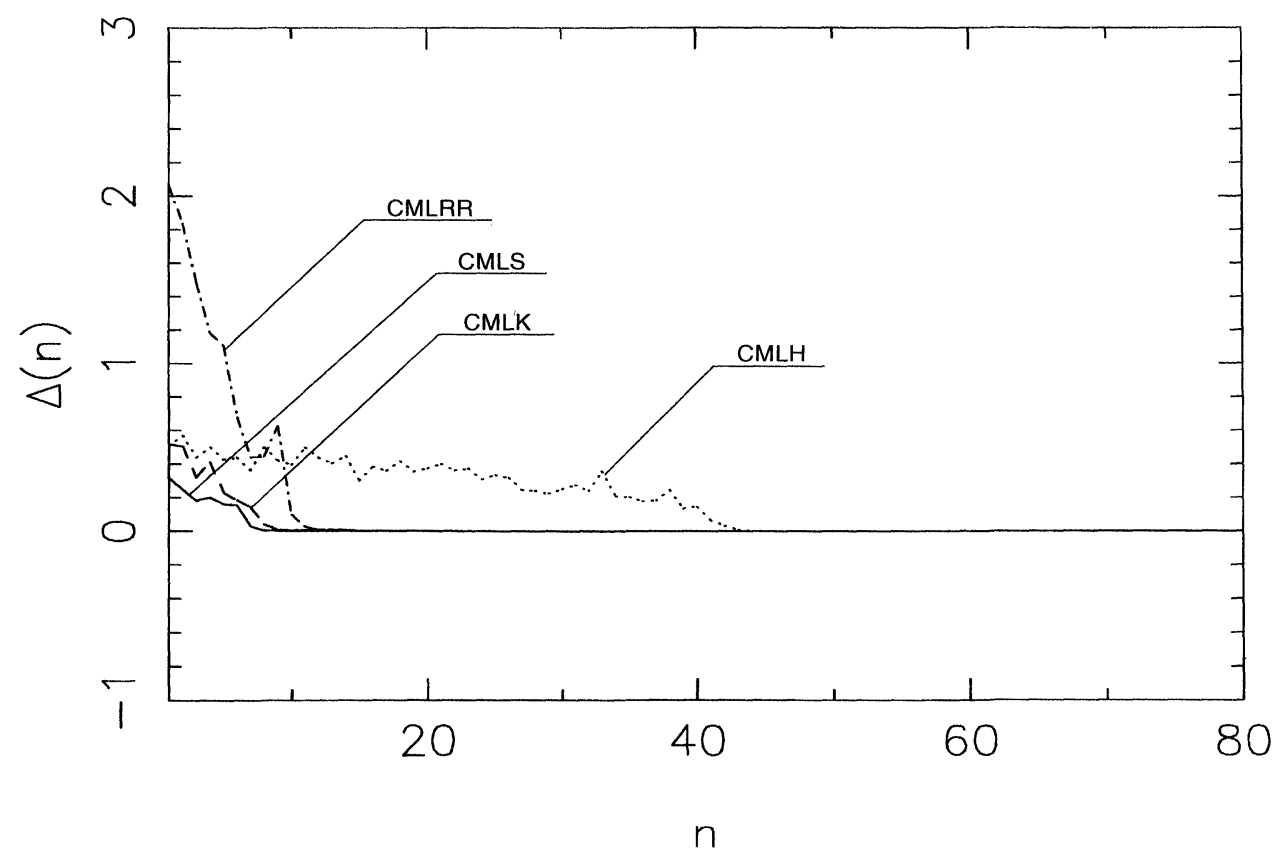

FIGURE 5(c)

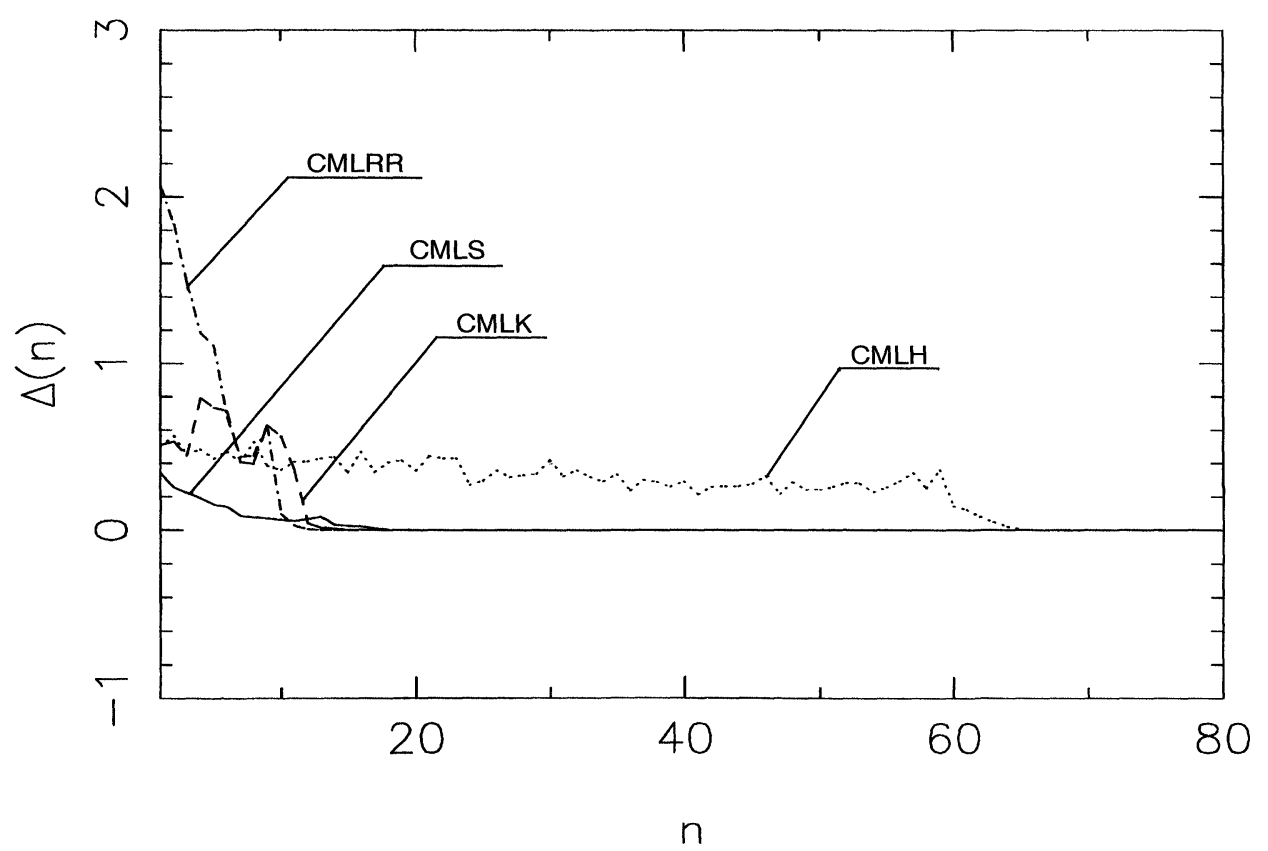

FIGURE 5(d)

FIGURE 5 A comparison of transition time $\Delta(n)$ for the CMLRR above, CMLS, CMLH and CMLK under the same NFF1 corresponding to Eqs. (15), (16), (18) and (21), respectively. CMLS: $L=50, N=2$ and $\epsilon=0.023$ and $a=4$. CMLH: $L=50, N=2$ and $\epsilon=0.8$ and $a=4$. CMLK: $L=50, N=L$ and $\epsilon=0.8$ and $a=4$. 


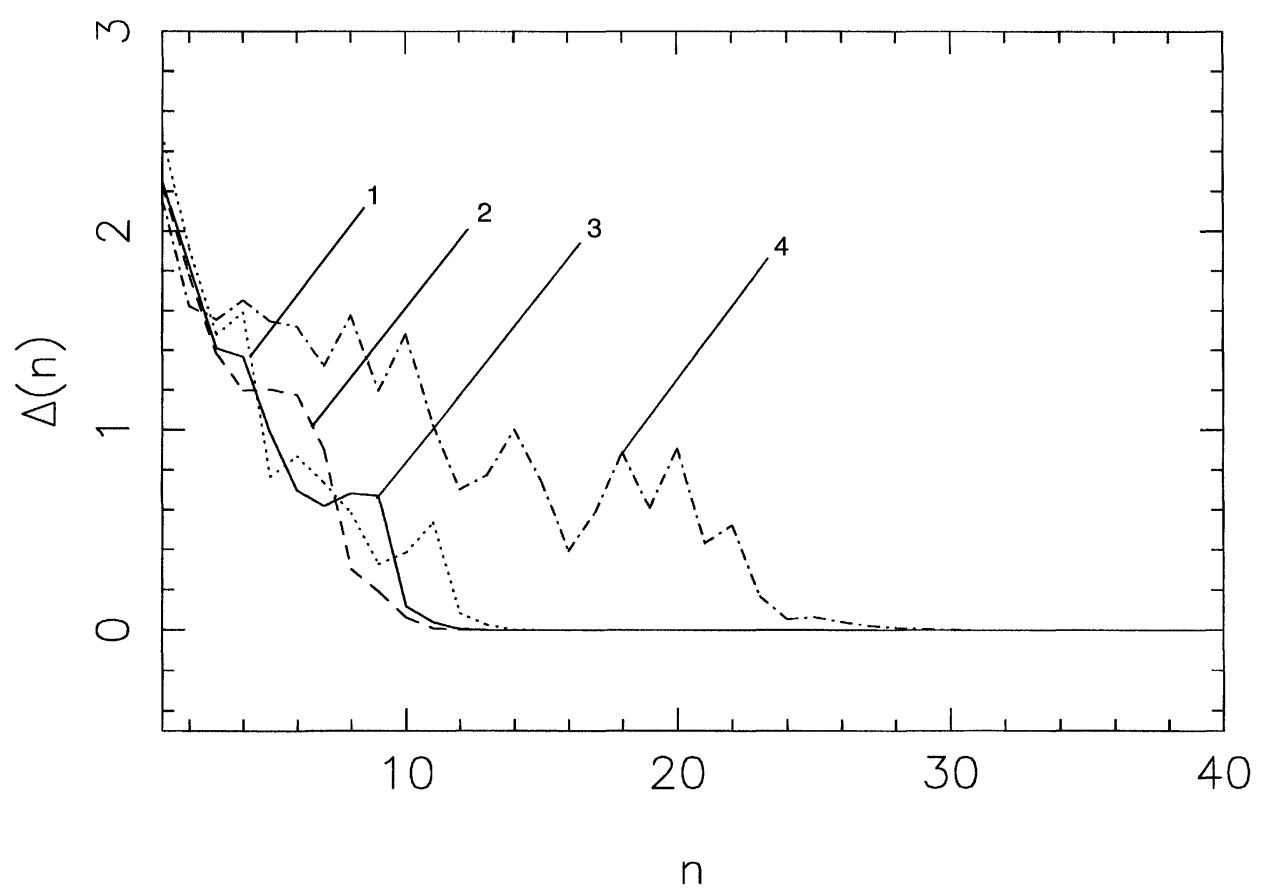

FIGURE 6(a)



FIGURE 6(b) 


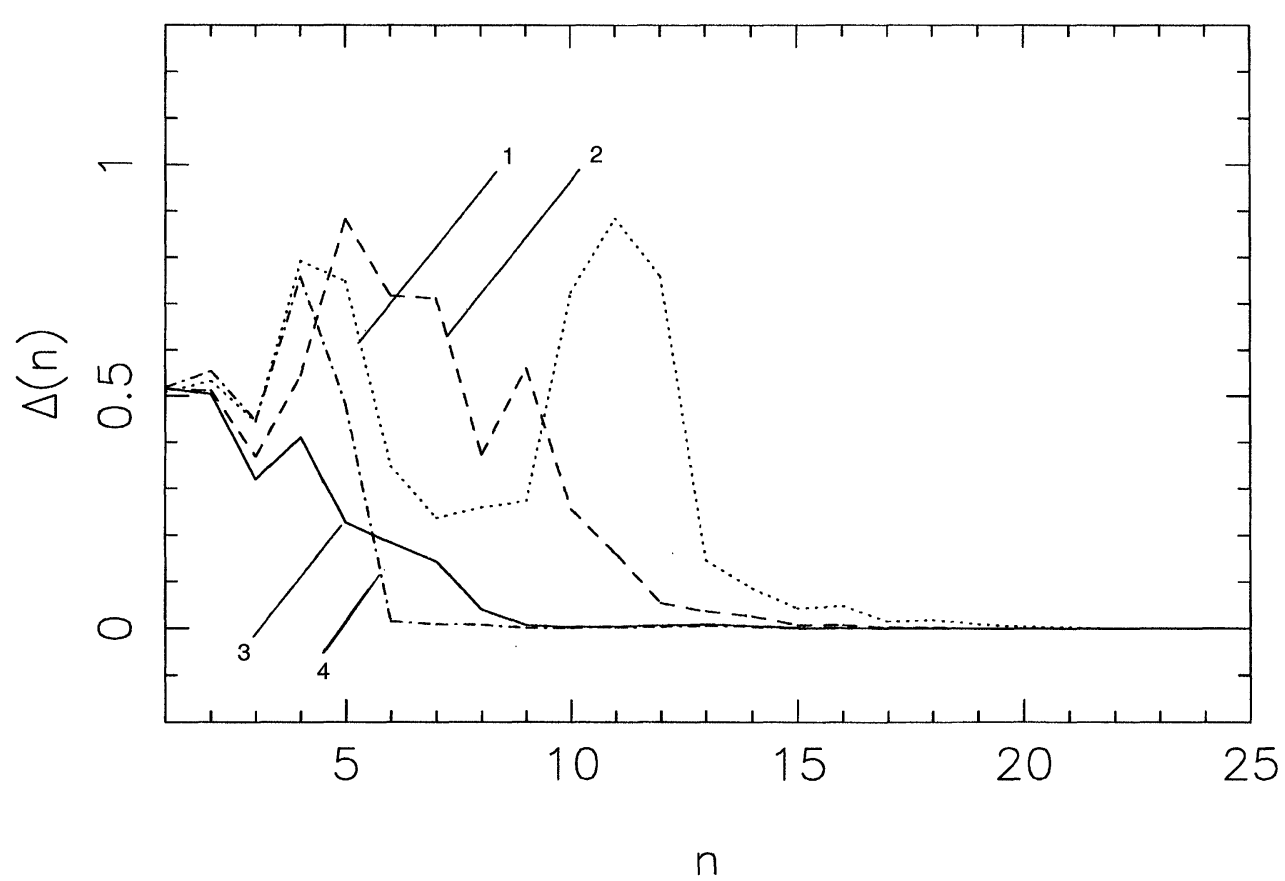

FIGURE 6(c)



FIGURE 6(d)

FIGURE 6 A comparison of transition time $\Delta(n)$ for stabilizing the fixed point for the (a) CMLRR, (b) CMLS, (c) CMLK and (d) CMLH by using four kinds of NFF1: Eq. (15) corresponds to curve-1, Eq. (22) to curve-2, Eq. (17) to curve-3 and Eq. (16) to curve-4. 
$N=2, \quad \epsilon=0.8$ ), controlled by four kinds of NFF1: Eq. (15) corresponds to curve-1, Eq. (22) to curve-2, Eq. (17) to curve-3 and Eq. (16) to curve-4, respectively. Clearly, these results show that any one of NFF1s Eqs. (15)-(22), can control the STC to the FP for the CMLR, CMLRR, CMLS, CMLH and CMLK effectively.

\subsection{Sporadic Feedback for Synchronization of STC}

So far we have considered control or synchronization of STC [23] in the CMLs applying the NFF1 to every site. This approach has successfully controlled/synchronized the CMLs for all values of $\epsilon$ in the interval $(0,1)$. It is pointed out that for certain ranges of values of $\epsilon, \gamma$ and $L$ it is not necessary to apply the NFF1 at every site for control/synchronization of STC. Sporadic feedback can also be implemented by using the NFF1. The success of sporadic feedback depends on the use of appropriate NFF1 and values of the system parameters. For example, two typical STCs in identical two CMLH with $(L=50, \epsilon=0.8)$ but different initial conditions and periodic boundary conditions are given in Figs. 7(a) and (b). Synchronization of the STC in Fig. 7(b) with Fig. 7(a) is achieved by applying the NFF1 Eq. (21) only at site 4 with $\gamma=0.5$ and at site 10 with $\gamma=2.5$, respectively; the corresponding transition times $\Delta(n)$ are shown in Fig. 7(c) and (d). Obviously the NFF1 can work well. It takes more transition time than that using feedback at each site but can save a lot of feedback signal in practice. Synchronization of periodic structure is also realized similarly.

\subsection{Conversion of the CMLs from the STC to Various Periodic Patterns}

It is also interesting that all CML systems of Eqs. (29)-(33) can be switched from the STC to the FP and various periodic patterns in time and in space by using suitable NFF1 with right



FIGURE 7(a) 




FIGURE 7(b)

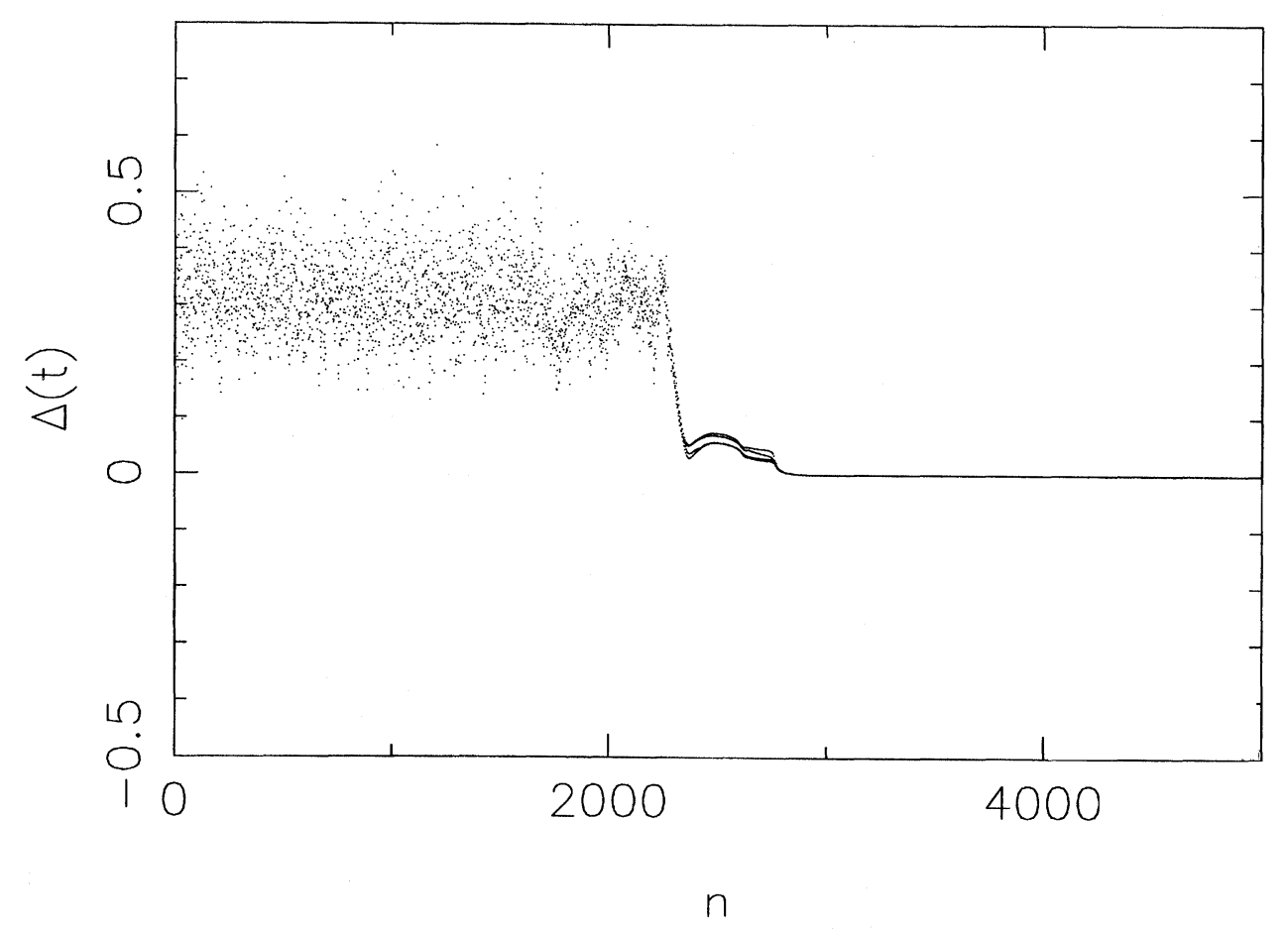

FIGURE 7(c) 


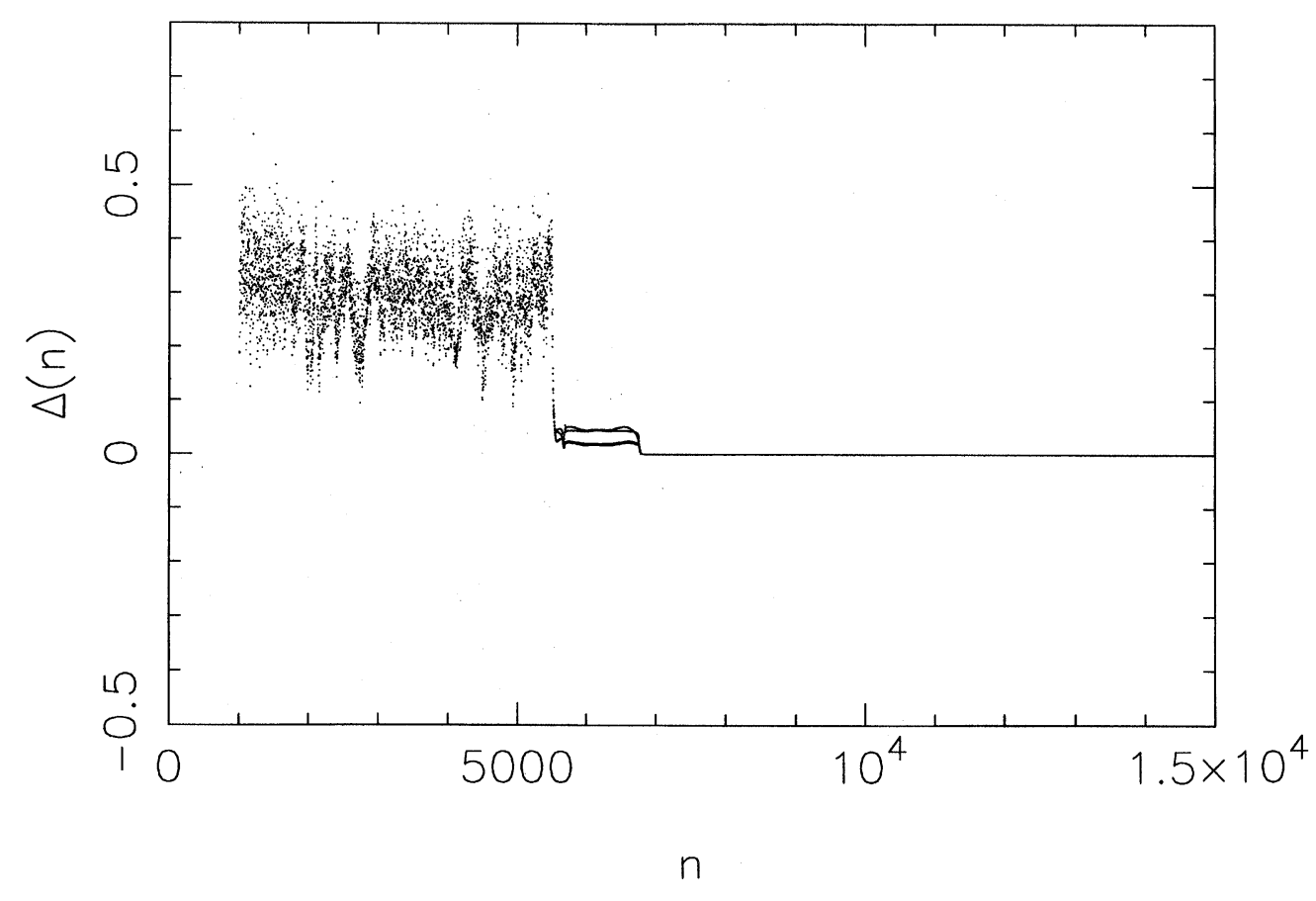

FIGURE 7(d)

FIGURE 7 Nonlinear feedback to sporadic site. (a) and (b) are typical STC in the CMLH, Eq. (31) with $L=50, N=2, \epsilon=0.8$ without any control. (c) Transition time $\Delta(n)$ of synchronization is implemented by using the sporadic NFF1 (16) with $K=3$, $\gamma=0.5$ at site 4 for the above CMLH. (d) The same as (c) but $\gamma=2.5$ at site 10 .

parameters. As an example, the conversions of the CMLRR with $L=50, N=20, \epsilon=0.96$ and $a=4$ from period-4, period-1 to two different FP are shown in Fig. 8 only if the values of $X_{\mathrm{d} n}$ are changed from $0.2,0.6,0.7$ and 0.75 in the same NFF1 of Eq. (22), respectively. We see from Fig. 8 that the oscillation wave forms of transition process correspond to four stabilized states. In fact, real fixed point for the CMLRR is $X_{\mathrm{d} n}=0.7428$. Therefore, $\Delta(n)$ vanishes and real fixed point is stabilized if $X_{\mathrm{d} n}=0.7428$. Here we ignored many similar conversion results. This kind of control is similar to control by the second kind NFF2 described in next subsection.

\subsection{Generating a Variety of New Spatiotemporal Patterns}

As mentioned above, the second kind NFF2 can work for controlling new STP if it is not easy or not necessary to know the solutions of UPP in the CML. In such a case, a rich variety of new UPP would be obtained in the CML by the NFF2. Figure 9 shows some typical controlled 3dimensional patterns generated by using NFF2, in which: (a) stationary spatial pattern in the CMLS with ( $L=50, \epsilon=0.0 .023, N=1, a=3.8)$ by using any one of the NFF2, above, such as $g_{n}(i)=\tanh \left(2 X_{n}(i)-1\right)\left(X_{n}(i)-1\right)$. However, it cannot be obtained by any linear feedback; (b) spatial pattern and period-1 in time for the CMLH with $(L=50, \epsilon=0.6, N=1, a=2)$ by using $g_{n}(i)=\left(2 X_{n}(i)-1\right)\left(5 X_{n}(i)-1\right)$; (c) spatial pattern and period-1 in time for the CMLR with ( $L=60, \epsilon=0.8, N=1, a=4)$ by using $g_{n}(i)=$ $0.5 X_{n}(i)\left(1.5 X_{n}(i)-1\right)$; (d) period-1 both in space and in time for the CMLH with $(L=60$, $\epsilon=0.8, \quad N=1, \quad a=2)$ by using $g_{n}(i)=0.5 X_{n}(i)$ $\left(1.25 X_{n}(i)-1\right)$. More complex patterns can also be obtained by using NFF2 but ignored here due 


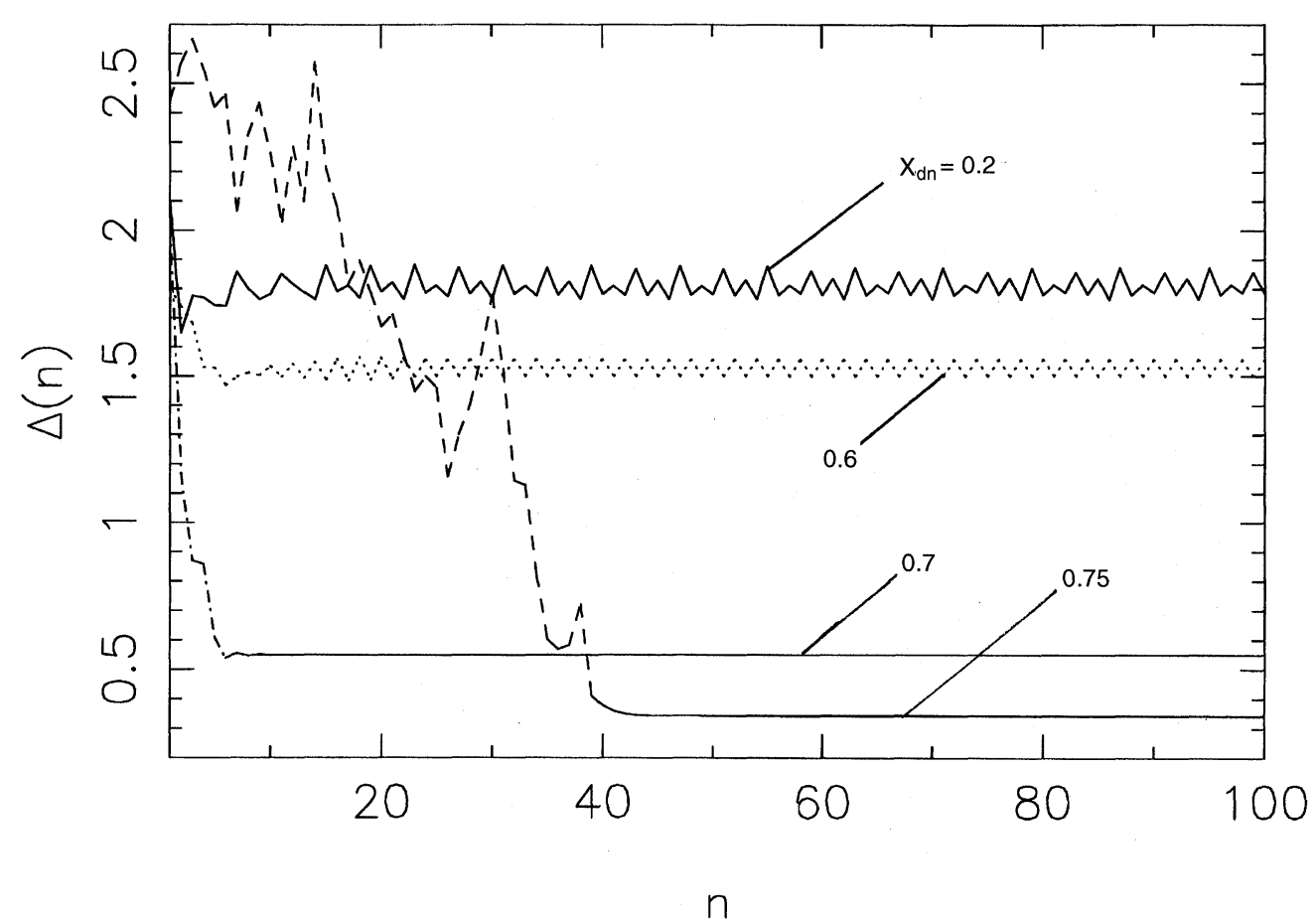

FIGURE 8 The conversion from STC to periods and fixed points for the CMLR with $L=50, N=10$ and $\epsilon=0.1$ and $a=4$. From period-4 (curve-1), period-1 (curve-2) to two fixed points (curve-3 and curve-4) when the values of $X_{\mathrm{d} n}$ are changed from $0.2,0.6,0.7$ to 0.75 in Eq. (22), respectively.

to limited space. It is seen from Fig. 9 that various spatiotemporal patterns in the CMLs may be generated by NFF2 which can be extended to nature and society similarly.

\section{DISCUSSIONS AND CONCLUSION}

We have answered the first question addressed in Introduction. In summary, The relationship between control and synchronization can be formulated in Eqs. (1)-(3) or Eqs. (8) and (9), which show that both cases have a common subject with different reference states for chosen purposes. They are two sides of common subject with different goal. The goal of chaos control is to stabilize a desired (or so-called goal, aim, target, reference) UPO or UPP embedded in the chaotic/ STC attractor, whereas the aim of synchronization may involve taking a chaotic/STC state as the reference state. Synchronism of STC/chaotic behavior is realized between two identical (or from the view point of generalized synchronization [24], different) systems with different initial conditions. However, synchronism is not only to realize synchronization of STC/chaotic behaviors between two identical systems but also to control a UPO/UPP. Therefore they can be viewed as the same mechanism with different goal states. Of course, sometimes they are rather different for various practical purposes. Research of synchronism of STC/chaos has been parallel to the study of control of STC/chaos. A good strategy that can be used for both cases is the linear and nonlinear feedback methods. We regard both cases as controlling chaos (including hyperchaos and spatiotemporal chaos): one for taming/suppressing chaos, the other for locking chaotic orbit to a desired state phase. 


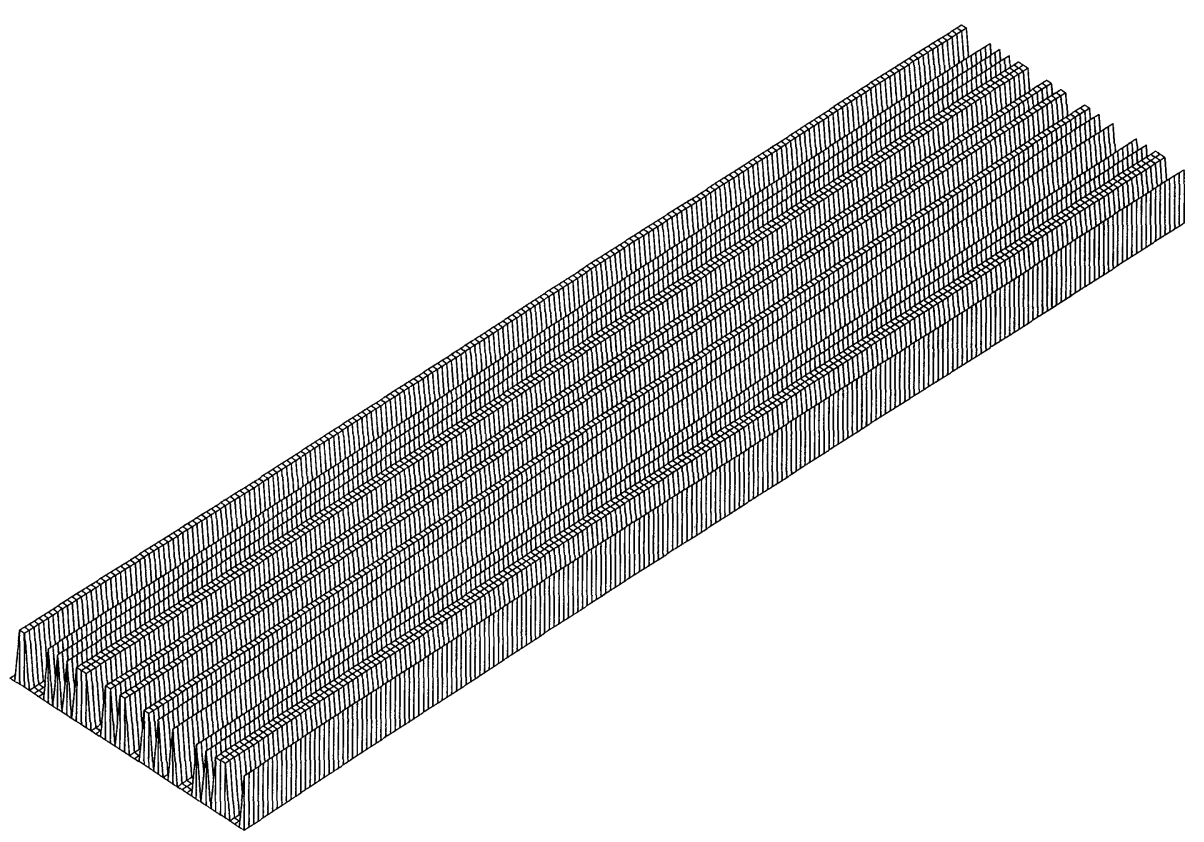

FIGURE 9(a)

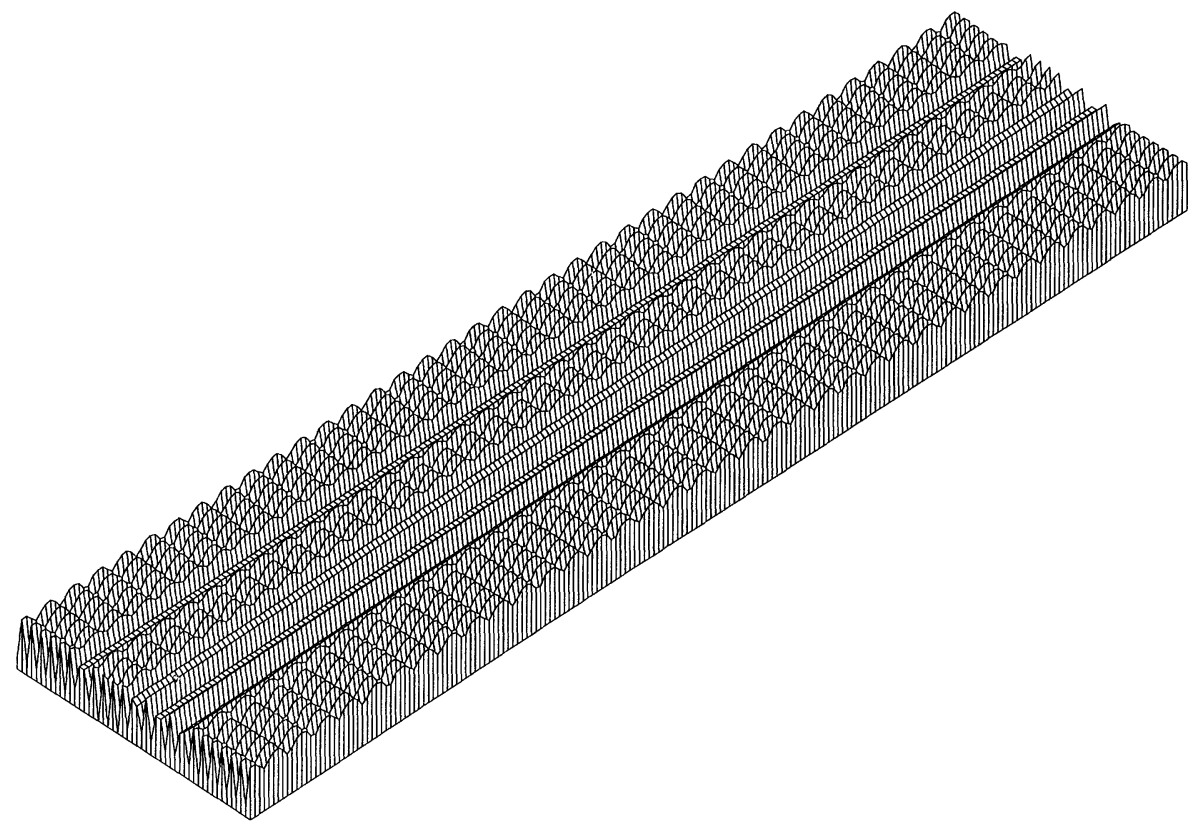

FIGURE 9(b) 


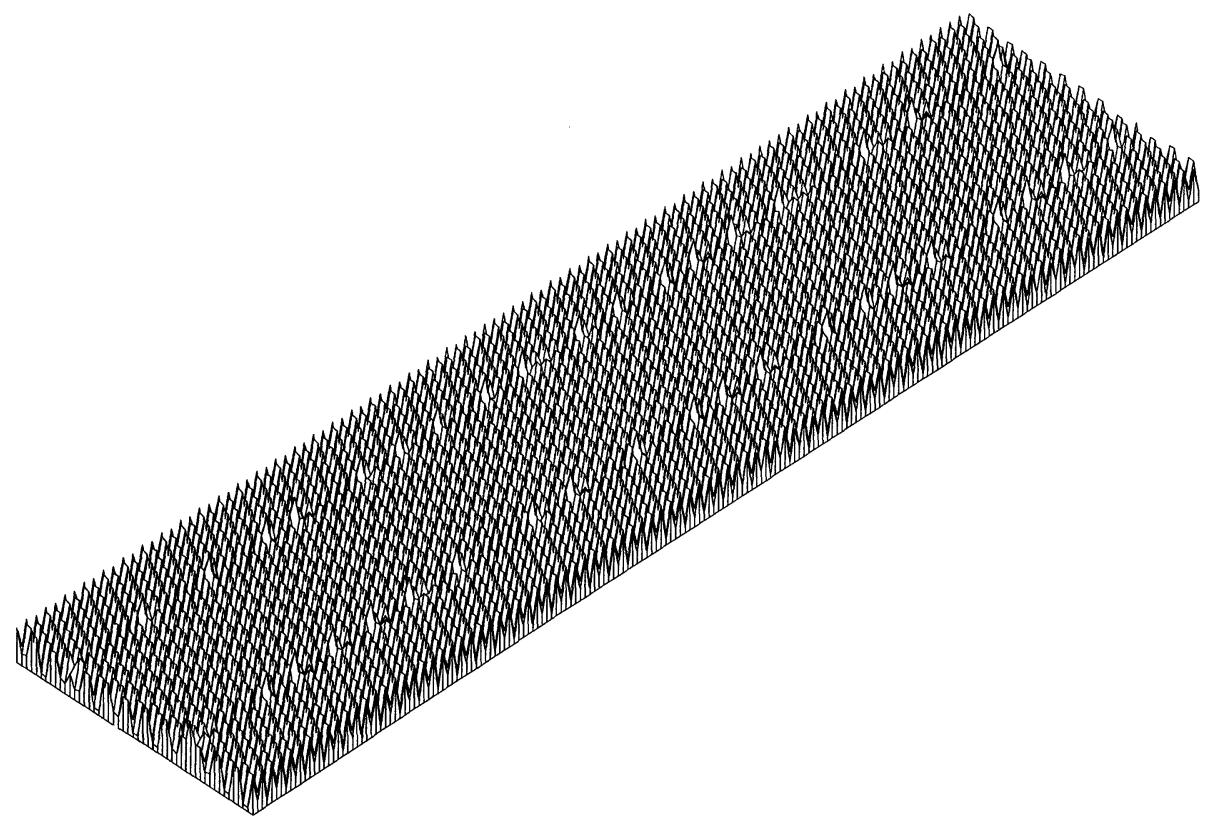

FIGURE 9(c)

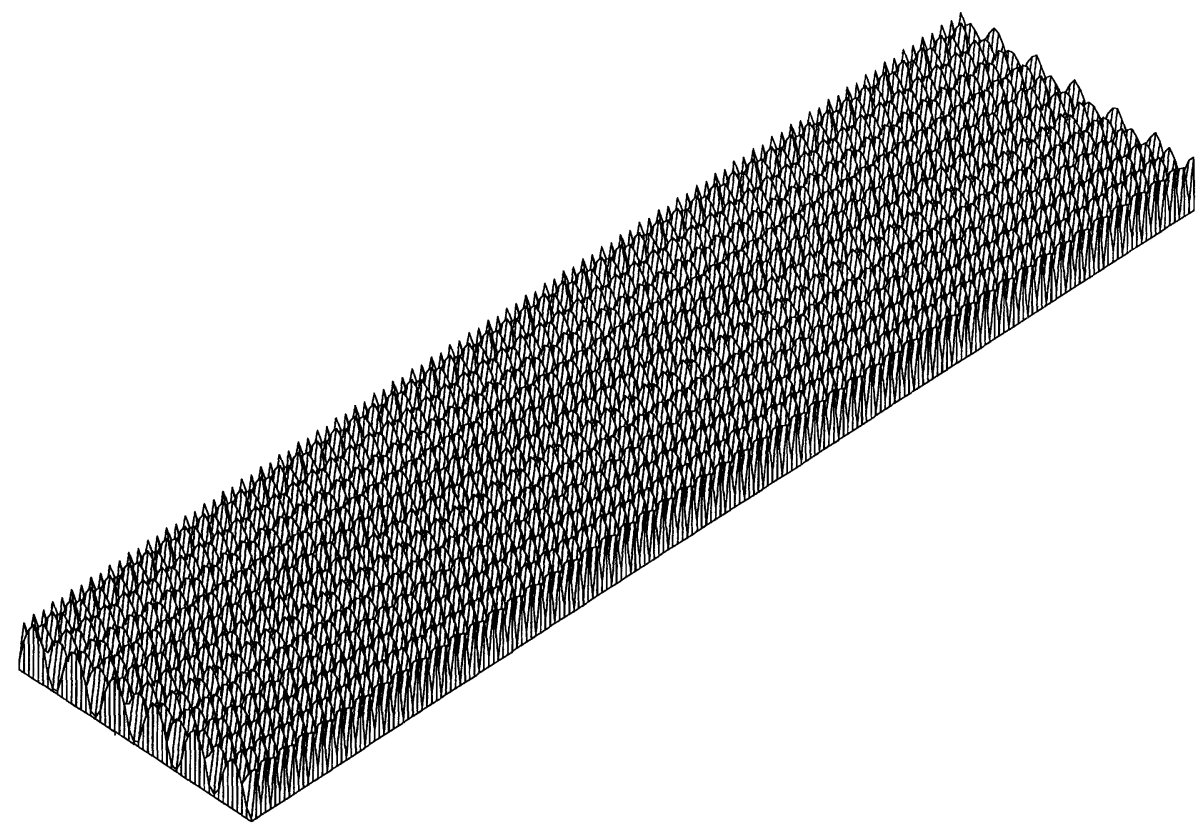

FIGURE 9(d)

FIGURE 9 Some new 3-dimensional patterns are generated by using NFF2. (a) Stationary spatial pattern in CMLS with $(L=50, \epsilon=0.0 .023, N=1, a=3.8)$ by using $g_{n}(i)=\left(2 X_{n}(i)-1\right)\left(X_{n}(i)-1\right)$. (b) Spatial pattern and period-1 in time for CMLH with ( $L=50, \epsilon=0.6, N=1, a=2)$ by using $g_{n}(i)=\left(2 X_{n}(i)-1\right)\left(5 X_{n}(i)-1\right)$. (c) Spatial pattern and period-1 in time for the CMLR with $(L=60, \epsilon=0.8, N=1, a=4)$ by using $g_{n}(i)=0.5 X_{n}(i)\left(1.5 X_{n}(i)-1\right)$. (d) Period-1 both in space and in time for the CMLH with $(L=60, \epsilon=0.8, N=1, a=2)$ by using $g_{n}(i)=0.5 X_{n}(i)\left(1.25 X_{n}(i)-1\right)$. 
Of course, we can also regard both cases as synchronizing chaos: one for synchronizing or stabilizing to desired UPO/UPP and the other for synchronizing chaotic/STC state between two identical systems with different initial conditions. Anyway, we may deal with both cases as a unified formulation above by various feedback strategies. Our recent work [23] and this work have shown that identical feedback method can be used for studying both control and synchronization of chaos/STC by using NFF, apart from different natures of the reference (targeted) state.

It can be seen from Figs. 1-7 that all of the NFF1s corresponding to Eqs. (15)-(22) work very well for control/synchronization of STC. Results with other values of $L, \nu$ and feedback to the dynamical variable for the CMLR, CMLRR, MLK, CMLS and CMLH with right parameters can achieve the control and synchronization of spatiotemporal chaos.

What is the mechanism for both cases by the NFFM? It is exciting to know development process for stabilizing UPP once the NFF signal is applied to the variables of the lattices. It is very similar to the process of reorganization in Ref. [4] and we observe synchronization itself to goal UPP in the CMLs. There are about three stages of evolution in general controlled by the NFF1. The first stage of evolution is still in chaos both in space and time when the feedback signal is just applied in the beginning, in which there are a few goal periodicities emerging through competing with different periodic patterns due to the role of the feedback signal $\mathbf{G}_{n}(i)$ and reorganization. When time evolution is going on, catastrophe phenomena occur in both time and space, where the goal UPP becomes winner, clearly preferred stable periodic pattern suddenly begins to synchronize with the goal pattern. Finally, it takes over the whole lattice when time $n$ goes on increasing. We also observe that the time scale of this transition of the goal time periodic pattern is found to be the same as that of the spatial transition to the spatial periodic pattern. This shows that the NFF signal speeds up simultaneously to synchronize to the goal pattern for the CML both in space and time. The above processes exhibit a possible mechanism of control of STC by the NFFM.

It is shown above that the nonlinear forms of the feedback function, such as main kinds of NFF1, Eqs. (15)-(22), are effective for controlling STC and synchronizing chaotic and hyperchaotic systems [23]. Obviously, the feedback scheme of Eqs. (1) and (3) and Eqs. (8) and (9) not only includes the approaches just mentioned but also has the flexibility of introducing possible new feedback functions and thus giving robustness to the method of feedback control, such as the active-passive decomposition scheme [24] and the approaches of Pecora and Carroll [25]. There are large classes of the NFF1 which may satisfy the above requested property. But there is no general method of constructing NFF1 so far. The NNF2 is suitable for generating new dynamics when desired UPP may not be known or it is not necessary and difficult to be found. Because of the robustness of the NFF method, it does not seem to be hard to find some feedback functions both of NFF1 and NFF2 for a given system by trial and error.

One can explore larger classes of nonlinear feedback functions to control or synchronize chaos and hyperchaos [23] as well as spatiotemporal chaos. We anticipate that a realization of the existence of such a flexibility will help design appropriate feedback control/synchronization in real experimental situations. It would be very interesting to see how these findings can be put to use in experiments. One area to which our findings may be directly relevant is the storage and retrieval of memory in neural networks. Another possible area of application is in secure communication - spatiotemporal chaotic or hyperchaotic signals are transmitted to mask a message and a synchronized receiver system is set to recover the message. Thus it may improve security and obtain a more efficient encoding of information.

In conclusion, the unified nonlinear feedback functional method is presented to study both 
control and synchronization of spatiotemporal chaos. We have clarified the method by the CML with five connection forms (CMLR, CMLRR, CMLK, CMLS and CMLH). We have not found any difficulty in controlling and synchronizing spatiotemporal chaos for the CMLs based on any large number of chaotic elements. Although our method has been applied to time continuous dynamical systems (as shown in hyperchaotic systems [23]), it is now applied to time discrete dynamical systems. It may be useful for considering social problems.

\section{Acknowledgements}

This work is supported by funding to Jin-Qing Fang from National Nuclear Industry Science Foundation of China, and China National Project of Science and Technology for Returned Student in Non-Education System. Fang wishes to thank the Department of Physics, the University of Lethbridge, for its hospitality. This research has also been supported by an NSERCC grant to M.K. Ali.

\section{References}

[1] W.L. Ditto, M.L. Spano and J.F. Lindner, Physica D, 86 (1995) 198; Fang Jin-Qing. Prog. in Phys. (in Chinese), 16 (1996) 1 and 16 (1996) 137.

[2] K. Kaneko, Prog. Theor. Phys., 72 (1984) 480; Physica D, 55 (1992) 368.

[3] A.P. Munuzuri, V. Perez-Munuzuri, M. Gomez-Gesteira, L.O. Chua and V. Perez-Villar, Inter. J. Bifur. and Chaos, 5 (1995) 17.

[4] Weiping Lu, Dejin $\mathrm{Yu}$ and R.G. Harrison, Phys. Rev. Lett., 76 (1996) 3316.
[5] R. Roy, T.W. Murphy, T.D. Maier, Z. Gills and E.R. Hunt, Phys. Rev. Lett., 68 (1992) 1259.

[6] G. Hu and K.F. He, Phys. Rev. Lett., 71 (1993) 3794; G. $\mathrm{Hu}, \mathrm{Z} . \mathrm{Qu}$ and $\mathrm{K} . \mathrm{He}$, Int. J. Bifur. and Chaos, 5 (1995) 901; J.H. Xiao, G. Hu and Z.L. Qu, Phys. Rev. Lett. 77 (1996) 4162.

[7] D. Auerbach, Phys. Rev. Lett., 72 (1994) 1184.

[8] J. Tang and H.H. Bau, Phys. Rev. Lett., 70 (1993) 1795.

[9] I. Aranson, H. Levine, and L. Tsimring, Phys. Rev. Lett., 72 (1994) 2561.

[10] J.A. Sepulchre and A. Babloyantz, Phys. Rev. E., 48 (1993) 945.

[11] G.A. Johnson, M. Locher and E.R. Hunt, Phys. Rev. E., 51 (1995) R1625.

[12] A. Hagberg, E. Meron, I. Rubinstein and B. Zaltzman, Phys. Rev. Lett., 76 (1996) 427.

[13] Y. Braiman, J.F. Lindner and W.L. Ditto, Nature, 378 (1995) 465

[14] F. Qin, E.E. Wolf and H-.C. Chang, Phys. Rev. Lett., 72 (1994) 1459.

[15] R. Martin, A.J. Scroggie, G.-L. Oppo and W.J. Firth, Phys. Rev. Lett., 77 (1996) 4007.

[16] A.L. Zheleznyak and L.O. Chua, Int. J. Bifur. and Chaos, 4 (1994) 639.

[17] J.H. Pen, E.J. Ding, M. Ding and W. Yang, Phys. Rev. Lett., 76 (1996) 904.

[18] T. Sinbrot, Advances in Physics, 44 (1995) 73.

[19] M.G. Rosenblum, A.S. Pikovsky and J. Kurths, Phys. Rev. Lett., 76 (1996) 1804.

[20] R. Shermer, A. Hubler and N. Packard, Phys. Rev. A, 43 (1991) 5642.

[21] V. Petrov and K. Showalter, Phys. Rev. Lett., 76 (1996) 3312.

[22] Proceedings of Workshop on Nonlinear Control and Control Chaos, Triest, Italy, 17-28 June 1996. Therein references, such as: Henk Nijmeijer, Hans Troger, and so on.

[23] M.K. Ali and Jin-Qing Fang, Phys. Rev. E, 55 (1997) 5285; M.K. Ali and Jin-Qing Fang, Discrete Dynamics in Nature and Society, 3 (1997); Jin-Qing Fang and M.K. Ali, Nucl. Sci. Tech., 8 (1997) 129; Jin-Qing Fang and M.K. Ali, Nucl. Sci. Tech., 8 (1997) 193.

[24] L. Kocarev and U. Parlitz, Phys. Rev. Lett., 74 (1995) 5028; Phys. Rev. Lett., 77 (1996) 2206; Phys. Rev. Lett., 76 (1996) 1816.

[25] L.M. Pecora and T.L. Carroll, Phys. Rev. Lett., 64 (1990) 821.

[26] P.M. Gade and R.E. Amrikar, Phys. Rev. E, 47 (1993) 143. 


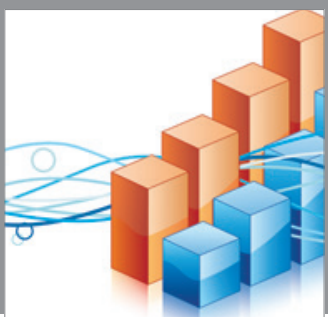

Advances in

Operations Research



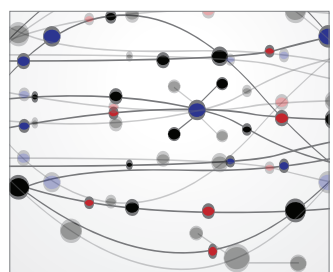

\section{The Scientific} World Journal
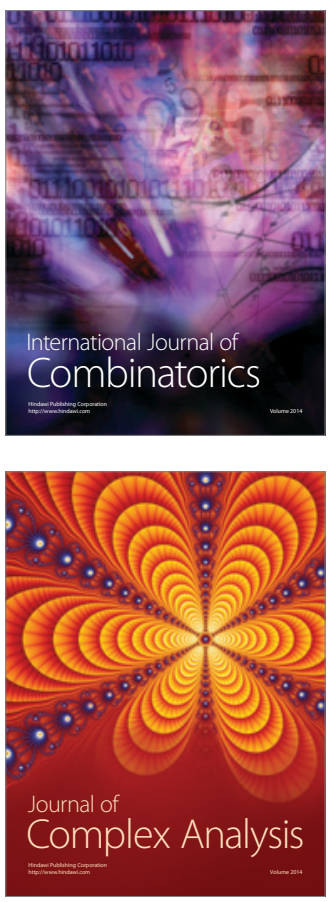

International Journal of

Mathematics and

Mathematical

Sciences
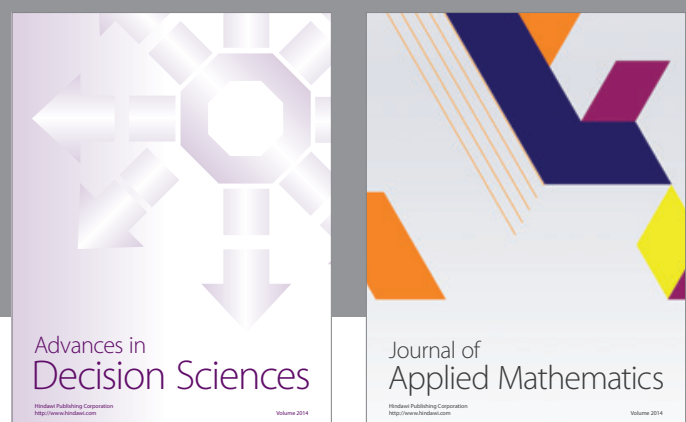

Journal of

Applied Mathematics
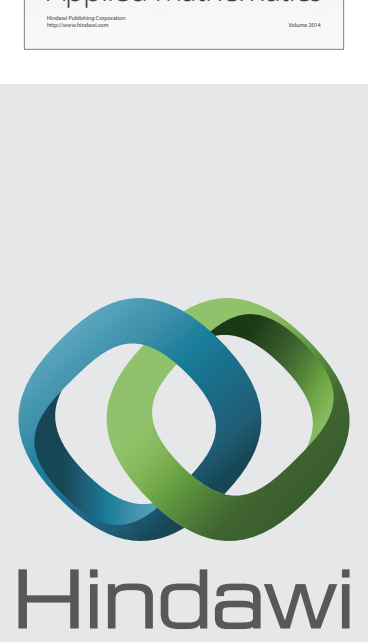

Submit your manuscripts at http://www.hindawi.com
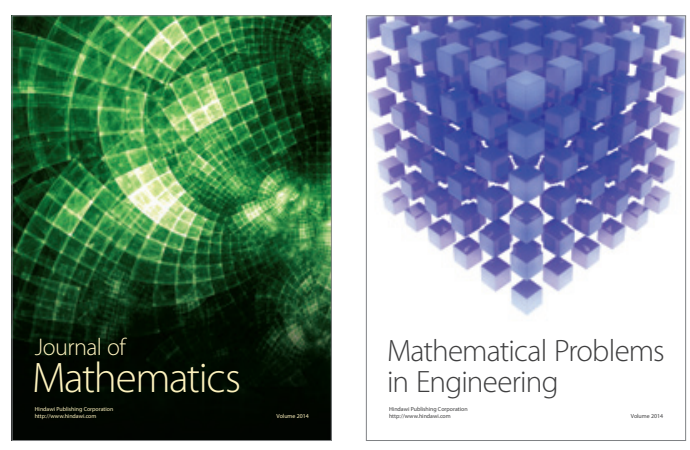

Mathematical Problems in Engineering
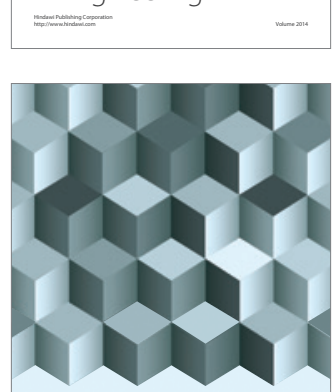

Journal of

Function Spaces
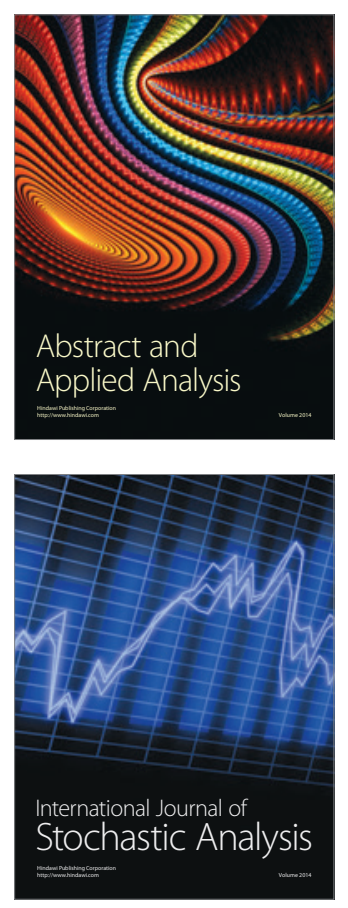

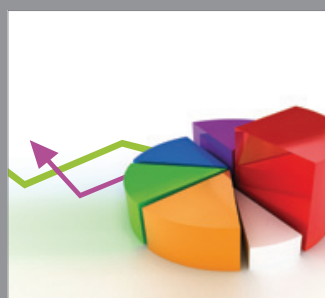

ournal of

Probability and Statistics

Promensencen
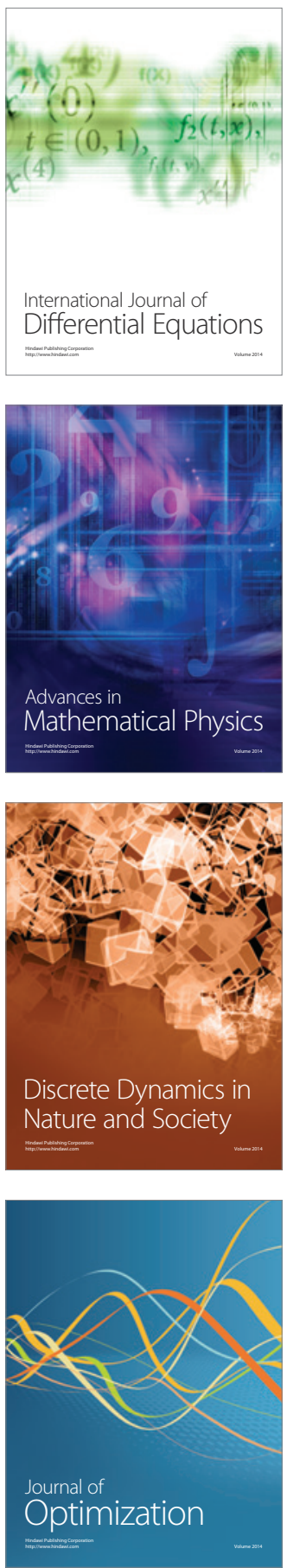